$\mathrm{UCLA} / 05 / \mathrm{TEP} / 2$

Columbia/Math/05

2005 January 24

\title{
TWO-LOOP SUPERSTRINGS V \\ Gauge Slice Independence of the N-Point Function ${ }^{1}$
}

\author{
Eric D'Hoker ${ }^{a}$ and D.H. Phong ${ }^{b}$ \\ ${ }^{a}$ Department of Physics and Astronomy \\ University of California, Los Angeles, CA 90095, USA \\ ${ }^{b}$ Department of Mathematics \\ Columbia University, New York, NY 10027, USA
}

\begin{abstract}
A systematic construction of superstring scattering amplitudes for $N$ massless NS bosons to two loop order is given, based on the projection of supermoduli space onto super period matrices used earlier for the superstring measure in the first four papers of this series. The one important new difficulty arising for the $N$-point amplitudes is the fact that the projection onto super period matrices introduces corrections to the chiral vertex operators for massless NS bosons which are not pure $(1,0)$ differential forms. However, it is proved that the chiral amplitudes are closed differential forms, and transform by exact differentials on the worldsheet under changes of gauge slices. Holomorphic amplitudes and independence of left from right movers are recaptured after the extraction of terms which are Dolbeault exact in one insertion point, and de Rham closed in the remaining points. This allows a construction of GSO projected, integrated superstring scattering amplitudes which are independent of the choice of gauge slices and have only physical kinematical singularities.
\end{abstract}

\footnotetext{
${ }^{1}$ Research supported in part by National Science Foundation grants PHY-01-40151 and DMS-02-45371.
} 


\section{Introduction}

In the papers [1, 2, 3, 4] of this series on superstring perturbation theory at two-loops, a gauge slice independent chiral superstring measure has been derived from first principles. The key procedure is a projection of supergeometries onto their super period matrices instead of onto the standard period matrices. This projection led to a new stress tensor correction as well as to new finite-dimensional determinant factors, both of which together rid the measure of all the ambiguities plaguing it in the past.

For the $N$-point function, it suffices to apply the same gauge-fixing procedure, after insertion of appropriate vertex operators. The one important subtlety is the emergence of some new corrections to the vertex operators, due to the deformation of complex structures inherent to the projection onto super period matrices.

The main goal of the present paper is to establish the gauge slice independence of the resulting $N$-point scattering amplitudes, when the corrections to the vertex operators are taken properly into account. Complete gauge slice independence will guarantee the absence of unphysical kinematical singularities in the full scattering amplitudes.

Underlying the gauge slice independence of the scattering amplitudes are two key results on the chiral amplitudes (or chiral blocks). The first is the fact that the chiral amplitudes are closed differential forms in the vertex operator insertion points, and transform by the addition of exact differentials on the worldsheet under change of slice. The second is a remarkable relation between superholomorphicity and holomorphicity: superholomorphic correlation functions, which are forms on products of super Riemann surfaces, descend to holomorphic forms on the products of the corresponding Riemann surfaces, modulo forms which are Dolbeault $\bar{\partial}$-exact in one variable and de Rham closed in the remaining variables. As a by-product, we obtain many new holomorphic sections of vector bundles over the moduli space of Riemann surfaces. These sections may be mathematically interesting in their own right.

We describe now our results in greater detail.

\subsection{Corrections to the Vertex Operators}

The key new subtlety in the evaluation of the $N$-point function originates from the fact that, in accordance with the projection onto the super period matrices $\hat{\Omega}_{I J}$, all vertex operators must be deformed to the complex structure defined by $\hat{\Omega}_{I J}$. Now the deformation of the correlation functions is accounted for by the same stress tensor insertion as in 1, 2, 3, 4] (see also the review [5]). However, a covariant amplitude must incorporate the volume form $d^{2 \mid 2} \mathbf{z} E(\mathbf{z})$ at the vertex position $\mathbf{z}$ on the super Riemann surface, and this volume form has to be deformed as well. Incorporating this volume form and its 
deformation, and integrating over the $\theta$ super-coordinate of $\mathbf{z}=(z, \theta)$, we obtain the following component expression for the full chiral vertex of NS massless bosons,

$$
\mathcal{V}(z ; \epsilon, k)=\mathcal{V}^{(0)}(z ; \epsilon, k)+\mathcal{V}^{(1)}(z ; \epsilon, k)+\mathcal{V}^{(2)}(z ; \epsilon, k)
$$

where

$$
\begin{aligned}
\mathcal{V}^{(0)}(z ; \epsilon, k) & =\epsilon^{\mu} d z\left(\partial_{z} x_{+}^{\mu}-i k^{\nu} \psi_{+}^{\mu} \psi_{+}^{\nu}\right)(z) e^{i k \cdot x_{+}(z)} \\
\mathcal{V}^{(1)}(z ; \epsilon, k) & =-\frac{1}{2} \epsilon^{\mu} d \bar{z} \chi_{\bar{z}}{ }^{+} \psi_{+}^{\mu}(z) e^{i k \cdot x_{+}(z)} \\
\mathcal{V}^{(2)}(z ; \epsilon, k) & =-\epsilon^{\mu} \hat{\mu}_{\bar{z}}^{z} d \bar{z}\left(\partial_{z} x_{+}^{\mu}-i k^{\nu} \psi_{+}^{\mu} \psi_{+}^{\nu}\right)(z) e^{i k \cdot x_{+}(z)}
\end{aligned}
$$

Here, $\mathcal{V}^{(0)}(z ; \epsilon, k)$ is the familiar vertex operator $\left(x_{+}(z)\right.$ and $\psi_{+}(z)$ denote respectively the effective chiral scalar boson and the chiral fermion), and $\mathcal{V}^{(1)}(z ; \epsilon, k)$ and $\mathcal{V}^{(2)}(z ; \epsilon, k)$ are corrections which depend both on the gravitino slice $\chi_{\bar{z}}{ }^{+}$and the Beltrami differential $\hat{\mu}_{\bar{z}}{ }^{z}$ for the passage from period matrix to super period matrix. In particular, the full vertex operators are gauge slice dependent.

We stress that $\mathcal{V}^{(0)}(z ; \epsilon, k)$ is a $(1,0)$ form, but $\mathcal{V}^{(1)}(z ; \epsilon, k)$ and $\mathcal{V}^{(2)}(z ; \epsilon, k)$ are $(0,1)$ forms, so that the full vertex operator $\mathcal{V}(z ; \epsilon, k)$ includes both $(1,0)$ and $(0,1)$ components. Although $\mathcal{V}^{(1)}(z ; \epsilon, k)$ and particularly $\mathcal{V}^{(2)}(z ; \epsilon, k)$ are the source of many complications, their omission would certainly lead to unacceptable gauge-dependent results for the final superstring amplitudes.

\subsection{The Chiral Amplitudes $\mathcal{B}[\delta]$}

Let $\mathcal{B}[\delta]$ denote the full chiral amplitude for the scattering of $N$ massless NS bosons, incorporating the chiral measure, ghost, and superghost contributions which were derived in [1, 2. The explicit expression for $\mathcal{B}[\delta]$ is given by ${ }^{1}$

$$
\begin{aligned}
\mathcal{B}[\delta]\left(z_{i} ; \epsilon_{i}, k_{i}, p_{I}\right)=\prod_{I \leq J} d \Omega_{I J} & \int \prod_{\alpha=1,2} d \zeta^{\alpha} \frac{\prod_{a=1}^{3} b\left(p_{a}\right) \prod_{\alpha=1}^{2} \delta\left(\beta\left(q_{\alpha}\right)\right)}{\operatorname{det} \Phi_{I J+}\left(p_{a}\right) \operatorname{det}\left\langle H_{\alpha} \mid \Phi_{\beta}^{*}\right\rangle} \\
& \times\left\langle Q\left(p_{I}\right) \exp \left\{\frac{1}{2 \pi} \int(\chi S+\hat{\mu} T)\right\} \prod_{j=1}^{N} \mathcal{V}_{j}\right\rangle
\end{aligned}
$$

The notations used here are those of [2]: the integration is over the odd super-moduli $\zeta^{\alpha}, \alpha=1,2 ; S$ and $T$ are the supercurrent and stress tensor respectively; $\Phi_{I J+}$ and $\Phi_{\alpha}^{*}$ are

\footnotetext{
${ }^{1}$ We use the new notation $\mathcal{B}[\delta]$, in order to distinguish this quantity from the chiral block $\mathcal{F}[\delta]$ derived in [6] and to be described in (2.8). The difference between the two is that the definition of $\mathcal{F}[\delta]$ does not include the effects of the measure factors $d^{2 \mid 2} \mathbf{z}_{\mathbf{i}} E\left(\mathbf{z}_{i}\right)$ at the vertex operators, while $\mathcal{B}[\delta]$ does. Also, the integration over the worldsheet $\Sigma$ will be abbreviated by $\int_{\Sigma} \rightarrow \int$, and the vertex operators by $\mathcal{V}_{i}=\mathcal{V}_{i}\left(z_{i}, \epsilon_{i}, k_{i}\right)$ when no confusion is expected to arise.
} 
super-holomorphic $3 / 2$ differentials; and $H_{\alpha}$ dual super-Beltrami differentials; $p_{I}$ are the internal loop momenta required by chiral splitting; and $Q\left(p_{I}\right)=\exp \left\{i p_{I}^{\mu} \oint_{B_{I}} d z \partial_{z} x_{+}^{\mu}(z)\right\}$.

The amplitude $\mathcal{B}[\delta]$ may be decomposed into the sum of a connected part $\mathcal{B}[\delta]^{(c)}$, and a disconnected part $\mathcal{B}[\delta]^{(d)}$,

$$
\begin{aligned}
\mathcal{B}[\delta] & =\mathcal{B}[\delta]^{(d)}+\mathcal{B}[\delta]^{(c)} \\
\mathcal{B}[\delta]^{(d)} & =d \mu_{2}[\delta]\left\langle Q\left(p_{I}\right) \prod_{i=1}^{N} \mathcal{V}_{i}^{(0)}\left(z_{i}, \epsilon_{i}, k_{i}\right)\right\rangle \\
\mathcal{B}[\delta]^{(c)} & =d \mu_{0}[\delta] \int \prod_{\alpha=1,2} d \zeta^{\alpha}\left(\mathcal{Y}_{1}+\mathcal{Y}_{2}+\mathcal{Y}_{3}+\mathcal{Y}_{4}+\mathcal{Y}_{5}\right)
\end{aligned}
$$

The disconnected part consists of the finite-dimensional determinants, and the self-contractions of the stress tensor $T$ and the supercurrents $S S$. It was already evaluated in [1]. The connected part is defined to be $\mathcal{B}[\delta]-\mathcal{B}[\delta]^{(d)}$. The prefactors $d \mu_{0}[\delta]$ and $d \mu_{2}[\delta]$ are components of the chiral measure $\mathcal{A}[\delta]$, defined and evaluated in [2],

$$
\begin{aligned}
\mathcal{A}[\delta] \prod_{I \leq J} d \hat{\Omega}_{I J} & =d \mu_{0}[\delta]+\zeta^{1} \zeta^{2} d \mu_{2}[\delta] \\
d \mu_{0}[\delta] & =\frac{\left\langle\prod_{a=1}^{3} b\left(p_{a}\right) \prod_{\alpha=1}^{2} \delta\left(\beta\left(q_{\alpha}\right)\right)\right.}{\operatorname{det} \omega_{I} \omega_{J}\left(p_{a}\right)} \prod_{I \leq J} d \hat{\Omega}_{I J} \\
d \mu_{2}[\delta] & =\frac{\vartheta[\delta](0, \hat{\Omega})^{4} \Xi_{6}[\delta](\hat{\Omega})}{16 \pi^{6} \Psi_{10}(\hat{\Omega})} \prod_{I \leq J} d \hat{\Omega}_{I J}
\end{aligned}
$$

The quantities $\mathcal{A}[\delta], d \mu_{0}[\delta]$ and $d \mu_{2}[\delta]$ are independent of the ghost insertion points $p_{a}$. While $d \mu_{2}[\delta]$ is also independent of $q_{1}$ and $q_{2}, d \mu_{0}[\delta]$ is not, but this dependence should disappear from the full amplitude which combines also the $\zeta$-dependent matter part. The various components $\mathcal{Y}_{i}, i=1, \cdots, 5$ are given by the following correlators,

$$
\begin{aligned}
& \mathcal{Y}_{1}=\frac{1}{8 \pi^{2}}\left\langle Q\left(p_{I}\right) \int \chi S \int \chi S \prod_{i=1}^{N} \mathcal{V}_{i}^{(0)}\right\rangle_{(c)} \\
& \mathcal{Y}_{2}=\frac{1}{2 \pi}\left\langle Q\left(p_{I}\right) \int \hat{\mu} T \prod_{i=1}^{N} \mathcal{V}_{i}^{(0)}\right\rangle_{(c)} \\
& \mathcal{Y}_{3}=\frac{1}{2 \pi} \sum_{i=1}^{N}\left\langle Q\left(p_{I}\right) \int \chi S \mathcal{V}_{i}^{(1)} \prod_{j \neq i}^{N} \mathcal{V}_{j}^{(0)}\right\rangle \\
& \mathcal{Y}_{4}=\frac{1}{2} \sum_{i \neq j}\left\langle Q\left(p_{I}\right) \mathcal{V}_{i}^{(1)} \mathcal{V}_{j}^{(1)} \prod_{l \neq i, j}^{N} \mathcal{V}_{l}^{(0)}\right\rangle \\
& \mathcal{Y}_{5}=\sum_{i=1}^{N}\left\langle Q\left(p_{I}\right) \mathcal{V}_{i}^{(2)} \prod_{j \neq i}^{N} \mathcal{V}_{j}^{(0)}\right\rangle
\end{aligned}
$$


The polarization vectors $\epsilon_{i}$ will be viewed as anti-commuting with themselves and with the differential 1-forms $d z_{i}$ and $d \bar{z}_{i}$, so that the vertices $\mathcal{V}_{i}$ commute with one another, and their ordering in the above correlators is arbitrary. This formal device was introduced and used extensively in [6, 7] and will be useful also here. Since all dependence on $\epsilon_{i}$ in the amplitudes is linear, this prescription reproduces the amplitudes. Finally, the $\delta$ dependence of $\mathcal{Y}_{1}, \cdots, \mathcal{Y}_{5}$ will be understood throughout.

All quantities above are expressed with respect to the superperiods $\hat{\Omega}_{I J}$. We recall the relations between the period matrix $\Omega_{I J}$, the super-period matrix $\hat{\Omega}_{I J}$ and the Beltrami differential $\hat{\mu}_{\bar{z}}{ }^{z}$ which provides the complex structure deformation between $\Omega_{I J}$ and $\hat{\Omega}_{I J}$,

$$
\begin{aligned}
\Omega_{I J}-\hat{\Omega}_{I J} & =\frac{i}{8 \pi} \int d^{2} z \int d^{2} w \omega_{I}(z) \chi_{\bar{z}}{ }^{+} S_{\delta}(z, w) \chi_{\bar{w}}{ }^{+} \omega_{J}(w) \\
& =i \int d^{2} z \hat{\mu}_{\bar{z}}{ }^{z} \omega_{I}(z) \omega_{J}(z)
\end{aligned}
$$

The vacuum expectation values in $\mathcal{Y}_{1}$ and $\mathcal{Y}_{2}$ are connected, as indicated by the subscript $\langle\cdots\rangle_{(c)}$, in the following sense. All self-contractions of $T$ are to be excluded. The contributions in which both $x_{+}$and $\psi_{+}$are contracted between the two supercurrents $S S$ are to be excluded. The contractions of only a single field between the two $S$-operators, with the remaining operators contracted elsewhere, however, are to be included in $\mathcal{Y}_{2}$.

The chiral blocks $\mathcal{B}[\delta]$ are 1 -forms (including both $(1,0)$ and $(0,1)$ components) in each vertex point $z_{i}$ with the following monodromy,

$$
\begin{aligned}
& \mathcal{B}[\delta]\left(z_{i}+\delta_{i j} A_{K}, \theta_{i} ; \epsilon_{i}, k_{i}, p_{I}\right)=\mathcal{B}[\delta]\left(z_{i}, \theta_{i} ; \epsilon_{i}, k_{i}, p_{I}\right) \\
& \mathcal{B}[\delta]\left(z_{i}+\delta_{i j} B_{K}, \theta_{i} ; \epsilon_{i}, k_{i}, p_{I}\right)=\mathcal{B}[\delta]\left(z_{i}, \theta_{i} ; \epsilon_{i}, k_{i}, p_{I}+\delta_{I K} k_{j}\right) .
\end{aligned}
$$

Thus they should be viewed as sections of a flat vector bundle over the moduli space of Riemann surfaces with $N$-punctures.

\subsection{The Structure of Chiral and Holomorphic Blocks}

It is a fundamental principle of string theory, reflecting the independence of left and rightmovers, that the superstring scattering amplitudes be realizable as hermitian pairings of holomorphic sections of vector bundles over the moduli space of Riemann surfaces with $N$ punctures. When $N>0$, an old puzzle of superstring perturbation theory is how to reconcile this holomorphicity requirement with the fact that its building blocks, namely the scalar superfield chiral amplitudes $\mathcal{F}[\delta]$, are only superholomorphic. For non-zero gravitino field $\chi_{\bar{z}}{ }^{+}$, the notion of superholomorphicity appears fundamentally different from the notion of holomorphicity. 
The vertex operator corrections $\mathcal{V}^{(1)}$ and $\mathcal{V}^{(2)}$ seemingly compound the problem: they are $(0,1)$-forms, and such forms do not even admit an intrinsic notion of holomorphicity, since their covariant derivative $\nabla_{\bar{z}}$ requires a connection.

Perhaps surprisingly, exactly the opposite is the case. The $(0,1)$ vertex operator correction terms provide precisely the mechanism for restoring holomorphicity to the string amplitudes, and this is in fact closely related to the ultimate gauge slice independence of the $N$-point function: with the $(0,1)$ correction terms, all the terms in the chiral amplitude $\mathcal{B}[\delta]$ which are not already tensor products of $(1,0)$-forms in all insertion points, combine into a sum $\sum_{i=1}^{N} \bar{\partial}_{i} \mathcal{S}_{i}[\delta]\left(z ; \epsilon, k, p_{I}\right)$ of terms which are Dolbeault $\bar{\partial}$-exact in one variable, and de Rham $d$-closed in the other variables. Upon completion of the Dolbeault $\bar{\partial}$-exact differential into a de Rham $d$-exact differential,

$$
d_{i} \mathcal{S}_{i}[\delta]\left(z ; \epsilon, k, p_{I}\right)=\bar{\partial}_{i} \mathcal{S}_{i}[\delta]\left(z ; \epsilon, k, p_{I}\right)+\partial_{i} \mathcal{S}_{i}[\delta]\left(z ; \epsilon, k, p_{I}\right),
$$

the de Rham $d$-exact terms $d_{i} \mathcal{S}_{i}[\delta]$ drop out of any physical amplitude, and the chiral amplitude $\mathcal{B}[\delta]$ is effectively replaced by the amplitude $\mathcal{H}[\delta]=\mathcal{B}[\delta]-\sum_{i=1}^{N} \partial_{i} \mathcal{S}_{i}[\delta]\left(z ; \epsilon, k, p_{I}\right)$, which is both holomorphic and gauge slice independent.

The precise statements are the following:

\section{(a) Closedness}

The forms $\mathcal{B}[\delta]$ are closed in each variable $z_{j} ;$

\section{(b) Slice-change by exact differentials on the worldsheet}

Under infinitesimal changes of either the gravitino slice $\chi$ or the Beltrami differential $\hat{\mu}$, the forms $\mathcal{B}[\delta]$ change by terms which are de Rham $d$-exact in one variable and de Rham $d$-closed in all other variables. ${ }^{2}$

$$
\mathcal{B}[\delta]\left(z ; \epsilon, k, p_{I}\right) \rightarrow \mathcal{B}[\delta]\left(z ; \epsilon, k, p_{I}\right)+\sum_{i=1}^{N} d_{i} \mathcal{R}_{i}[\delta]\left(z ; \epsilon, k, p_{I}\right)
$$

Specifically, $\mathcal{R}_{i}[\delta]$ is a form of weight $(0,0)$ in $z_{i}$, and a form of weight $(1,0) \oplus(0,1)$, which is de Rham closed, in each $z_{j}$ for $j \neq i$; Finally, $\mathcal{R}_{i}[\delta]$ has the same monodromy as $\mathcal{B}[\delta]$.

\section{(c) Holomorphicity}

There exist forms $\mathcal{S}_{i}[\delta]$ with properties similar to the ones listed for $\mathcal{R}_{i}[\delta]$ so that

$$
\mathcal{B}[\delta]\left(z ; \epsilon, k, p_{I}\right)-\sum_{i=1}^{N} \bar{\partial}_{i} \mathcal{S}_{i}[\delta]\left(z ; \epsilon, k, p_{I}\right) \quad \in \quad \otimes_{j=1}^{N} T_{1,0}^{z_{j}}(\Sigma) .
$$

\footnotetext{
${ }^{2}$ Henceforth, we shall denote the dependence of the blocks on $\left(z_{i} ; \epsilon_{i}, k_{i}, p_{I}\right)$ simply by $\left(z ; \epsilon, k, p_{I}\right)$.
} 
Thus the forms $\mathcal{B}[\delta]$ can be rewritten as

$$
\mathcal{B}[\delta]\left(z ; \epsilon, k, p_{I}\right)=\mathcal{H}[\delta]\left(z ; \epsilon, k, p_{I}\right)+\sum_{i=1}^{N} d_{i} \mathcal{S}_{i}[\delta]\left(z ; \epsilon, k, p_{I}\right)
$$

with $\mathcal{H}[\delta]\left(z ; \epsilon, k, p_{I}\right)$ having the same monodromy as $\mathcal{B}[\delta]$, of weight $(1,0)$ and holomorphic

in each variable $z_{i}$ with respect to the complex structure defined by $\hat{\Omega}_{I J}$, and away from coincident points $z_{i}=z_{j}$ for $i \neq j$.

\section{(d) Structure of the Scattering Amplitudes}

The properties (a) and (b) imply immediately the invariance of the integrals

$$
\int d p_{I} \int_{\Sigma^{N}} \mathcal{B}[\delta]\left(z ; \epsilon, k, p_{I}\right) \wedge \overline{\mathcal{B}^{\prime}[\bar{\delta}]\left(z ; \epsilon, k, p_{I}\right)}
$$

under $\chi$ and $\hat{\mu}$ changes, thus establishing the desired gauge-slice invariance. Here, the integration is over $N$ copies of the worldsheet parametrized by coordinates $z_{i}, i=1, \cdots, N$.

The property (c) shows that the superstring amplitudes can be expressed as integrals over internal momenta $p_{I}$ and over the moduli space $\mathcal{M}_{2}$ of genus 2 Riemann surfaces of a Hermitian pairing of holomorphic forms,

$$
\sum_{\delta, \bar{\delta}} \mathcal{C}_{\delta, \bar{\delta}} \int d p_{I} \int_{\mathcal{M}_{2}} \int_{\Sigma^{N}} \mathcal{H}[\delta]\left(z ; \epsilon, k, p_{I}\right) \wedge \overline{\mathcal{H}^{\prime}[\bar{\delta}]\left(z ; \epsilon, k, p_{I}\right)}
$$

thus restoring in this manner the basic principle of independence of left and right movers, as is essential to the formulations of both the Type II and Heterotic strings. The coefficients $C_{\delta, \bar{\delta}}$ reflect the GSO projection, and their choices are governed by invariance under the modular group $S p\left(4, \mathbf{Z}_{2}\right)$.

\subsection{Summary of the Algorithm for the $N$-point Function}

In conclusion, we have now a straightforward procedure for evaluating the $N$-point function, consisting of the following three steps:

- Deform the complex structure from the period matrix to the super period matrix. This requires a choice of gravitino slice $\chi_{\bar{z}}{ }^{+}$and Beltrami differential $\hat{\mu}_{\bar{z}}{ }^{z}$;

- Insert the full vertex operator $\mathcal{V}$ for the emission of each NS state. This vertex operator includes the gauge-dependent corrections $\mathcal{V}^{(1)}$ and $\mathcal{V}^{(2)}$. This results in the chiral amplitude $\mathcal{B}[\delta]$ of (1.4) - (1.6); 
- Extract the Dolbeault $\bar{\partial}$-exact forms and complete them into de Rham $d$-exact forms. These de Rham exact terms cancel out of the superstring amplitude, upon pairing left and right movers. The remaining forms are pure $(1,0)$ and holomorphic forms in each insertion point $z_{i}$, away from $z_{i}=z_{j}$ for $i \neq j$.

This procedure can be implemented for each spin structure $\delta$, and the gauge-fixed $N$ point function is obtained by pairing the holomorphic forms of the left movers with the anti-holomorphic forms of the right movers, and summing independently over the spin structures $\delta, \tilde{\delta}$. The gauge-fixed $N$-point function is then guaranteed to be independent of all choices of gauge slice ${ }^{3}$.

In the present paper, we shall establish the properties (a) and (b) of $\S 1.3$, which are the ones needed for gauge-slice independence of the $N$-point amplitude. The explicit evaluation of the $N$-point function and the derivation of the holomorphic blocks $\mathcal{H}[\delta]$ will be given in forthcoming publications [8, 9].

Finally, we should add that there is an extensive literature on the calculation of $N$-point functions for the superstring [10, 11, 12 in the RNS formulation and more specifically on the calculation of two-loop amplitudes [10, 13, 14, 15]. We shall discuss some of these works at greater length in the forthcoming paper [8] dealing with non-renormalization theorems and explicit formulas for the 4-point function. We note that a different, spacetime supersymmetric, approach to superstring amplitudes was pursued in [16] and more recently in [17].

\footnotetext{
${ }^{3}$ The sums over spin structures can also be carried out first, which can simplify the task of extracting the Dolbeault $\bar{\partial}$-exact forms (see 8 for the case of $N \leq 4$ ). The procedure for extracting Dolbeault $\bar{\partial}$-exact forms for fixed $\delta$ is much more complicated, but it produces more holomorphic sections.
} 


\section{Chiral Splitting and Chiral Blocks}

Our considerations start from two earlier results: first, the superstring measure derived in [1, 2]; second, the chiral splitting theorem obtained in [6] for the correlation functions of scalar superfields. We review briefly these results. For background on two-dimensional supergeometry, we refer to [2, 17, 18].

\subsection{Chiral Splitting}

Let $\Sigma$ be the worldsheet, which is a surface of genus $h$ at perturbative order $h$, equipped with a spin structure $\delta$ and a supergeometry $\left(E_{M}{ }^{A}, \Omega_{M}\right)$ satisfying the Wess-Zumino torsion constraints [7, 18. In the RNS formulation, superstring propagation is described by 10 scalar superfields $X^{\mu}(\mathbf{z}, \overline{\mathbf{z}})=x^{\mu}(z, \bar{z})+\theta \psi_{+}^{\mu}(z, \bar{z})+\bar{\theta} \psi_{-}^{\mu}(z, \bar{z})+i \theta \bar{\theta} F^{\mu}(z, \bar{z})$. The field $F^{\mu}$ is auxiliary and its effects are compensated by contact terms, as was shown in [6]. The generating vertex for massless NS bosons is [6, 19,

$$
V(\mathbf{z}, \overline{\mathbf{z}} ; \epsilon, \bar{\epsilon}, k)=\exp \left(i k^{\mu} X^{\mu}+\epsilon^{\mu} \mathcal{D}_{+} X^{\mu}+\bar{\epsilon}^{\mu} \mathcal{D}_{-} \bar{X}^{\mu}\right)(\mathbf{z}, \overline{\mathbf{z}})
$$

where $k^{2}=k \cdot \epsilon=k \cdot \bar{\epsilon}=0$ and $\mathcal{D}_{ \pm}$are covariant derivatives with respect to the supergeometry $\left(E_{M}{ }^{A}, \Omega_{M}\right)$. The polarization vectors $\epsilon_{i}$ are viewed as anti-commuting variables, so that $V$ is Grassmann even, a formal device first introduced in [6, 7]. The physical vertex operators are recovered from the generating vertex by retaining in $V(\mathbf{z}, \overline{\mathbf{z}} ; \epsilon, \bar{\epsilon}, k)$ only the contribution which is linear in $\epsilon_{i}$, linear in $\bar{\epsilon}_{i}$ and integrating that part against the vertex measure $d^{2 \mid 2} \mathbf{z}_{i} E\left(\mathbf{z}_{i}\right)$. The chiral splitting theorem (see specifically (4.28) and (5.4) in [6]; and also [20, 21]) asserts that ${ }^{4}$

$$
\left\langle\prod_{i=1}^{N} V\left(\mathbf{z}_{i}, \overline{\mathbf{z}}_{i} ; \epsilon_{i}, \bar{\epsilon}_{i}, k_{i}\right)\right\rangle_{X}=\int d p_{I}\left|\left\langle Q\left(p_{I}\right) \exp \left(\frac{1}{2 \pi} \int \chi S\right) \prod_{i=1}^{N} W\left(\mathbf{z}_{i} ; \epsilon_{i}, k_{i}\right)\right\rangle_{+}\right|^{2}
$$

Here, all contractions in the correlator on the right hand side are to be carried out with the following effective rules ${ }^{5}$ for the chiral fields $x_{+}(z)$ and $\psi_{+}(z)$,

$$
\begin{aligned}
& \left\langle x_{+}(z) x_{+}(w)\right\rangle_{+}=-\ln E(z, w), \\
& \left\langle\psi_{+}(z) \psi_{+}(w)\right\rangle_{+}=-S_{\delta}(z, w),
\end{aligned}
$$

where $E(z, w)$ and $S_{\delta}(z, w)$ are respectively the prime form and the Szegö kernels with respect to the complex structure of $g_{m n}$ (equivalently, of $\Omega_{I J}$ ). Also,

$$
Q\left(p_{I}\right)=\exp \left\{i p_{I}^{\mu} \oint_{B_{I}} d z \partial_{z} x_{+}^{\mu}(z)\right\}
$$

\footnotetext{
${ }^{4}$ Throughout, we shall use the following abbreviations for supercurrent and stress tensor insertions, $\int \chi S \equiv \int d^{2} z \chi_{\bar{z}}{ }^{+} S(z)$ and $\int \hat{\mu} T \equiv \int d^{2} z \hat{\mu}_{\bar{z}} z T(z)$.

${ }^{5}$ This instruction is indicated explicitly by the + subscript to the correlator $\langle\cdots\rangle_{+}$, but will be omitted whenever no confusion is expected to occur.
} 
and $W$ is a chiral worldsheet superspace generating vertex,

$$
W(\mathbf{z} ; \epsilon, k)=\exp \left\{i k^{\mu}\left(x_{+}^{\mu}+\theta \psi_{+}^{\mu}\right)(z)+\epsilon^{\mu}\left(\psi_{+}^{\mu}+\theta \partial_{z} x_{+}^{\mu}\right)(z)\right\}
$$

For recent applications of the subtleties of chiral splitting, see [22].

Next, we need to split chirally the integrals over all supergeometries

$$
\mathbf{A}[\delta]=\int D E_{M}{ }^{A} D \Omega_{M} \delta(T) \int \prod_{i=1}^{N} d^{2 \mid 2} \mathbf{z}_{i} E\left(\mathbf{z}_{i}\right)\left\langle\prod_{i=1}^{N} V\left(\mathbf{z}_{i}, \overline{\mathbf{z}}_{i} ; \epsilon_{i}, \bar{\epsilon}_{i}, k_{i}\right)\right\rangle_{X},
$$

after suitable gauge-fixing. We follow the same gauge-fixing procedure as in 2], §3. Henceforth, we assume that the genus of the worldsheet $\Sigma$ is $h=2$, and we fix a canonical homology basis $A_{I}, B_{I}$, \# $\left(A_{I} \cap B_{J}\right)=\delta_{I J}$. Supermoduli space is then of dimension $(3 \mid 2)$, to be parametrized by the even coordinates $\hat{\Omega}_{I J}, 1 \leq I \leq J \leq 2$, and by two odd coordinates $\zeta^{\alpha}, 1 \leq \alpha \leq 2$ [23]. As in [2], §3, we choose metrics $\hat{g}_{m n}$ whose period matrices are $\hat{\Omega}_{I J}$. For any two independent gravitino gauge slice functions $\hat{\chi}_{\alpha}$, set $\chi=\sum_{\alpha=1}^{2} \zeta^{\alpha} \hat{\chi}_{\alpha}$, and define $\Omega_{I J}$ by the equation (1.7), with $\chi_{\alpha}$ replaced by $\hat{\chi}_{\alpha}$. Next, choose metrics $g_{m n}$ whose period matrices are $\Omega_{I J}$. We may assume that $g_{m n}$ and $\hat{g}_{m n}$ differ only by terms of second order in $\zeta^{\alpha}$, so that $\hat{\chi}_{\alpha}$ can also be viewed as gravitino fields $\chi_{\alpha}$ with respect to $g_{m n}$. Then the supergeometry $\left(g_{m n}, \chi=\sum_{\alpha=1}^{2} \zeta^{\alpha} \chi_{\alpha}\right)$ admits $\hat{\Omega}_{I J}$ as its super period matrix, and defines a (3|2)-dimensional slice $\mathcal{S}$ for supermoduli space. The gauge-fixing method of [2], §3 gives now

$$
\mathbf{A}[\delta]=\int d p_{I} \int_{\mathcal{M}_{2}} \int \prod_{i=1}^{N} d^{2 \mid 2} \mathbf{z}_{i} E\left(\mathbf{z}_{i}\right)\left|\prod_{I \leq J} d \hat{\Omega}_{I J} \prod_{\alpha=1}^{2} d \zeta^{\alpha} \mathcal{F}[\delta]\left(z_{i}, \theta_{i} ; \epsilon_{i}, k_{i}, p_{I}\right)\right|^{2}
$$

with

$$
\mathcal{F}[\delta]=\frac{\left\langle\prod_{a=1}^{3} b\left(p_{a}\right) \prod_{\alpha=1}^{2} \delta\left(\beta\left(q_{\alpha}\right)\right)\right\rangle}{\operatorname{det} \Phi_{I J+}\left(p_{a}\right) \operatorname{det}\left\langle H_{\alpha} \mid \Phi_{\beta}^{*}\right\rangle}\left\langle Q\left(p_{I}\right) \exp \left(\frac{1}{2 \pi} \int \chi S\right) \prod_{i=1}^{N} W\left(\mathbf{z}_{i}, \epsilon_{i}, k_{i}\right)\right\rangle_{+}(g)
$$

Here $p_{a}$ and $q_{\alpha}$ are arbitrary points on the surface $\Sigma, b(z), \beta(z)$ are ghost and superghost fields, and the total supercurrent $S(z)$ is given by the sum of the matter and ghost supercurrents, $S(z)=S_{m}(z)+S_{g h}(z)$, where

$$
\begin{aligned}
S_{m}(z) & =-\frac{1}{2} \partial_{z} x_{+}^{\mu} \psi_{+}^{\mu}(z) \\
S_{g h}(z) & =\frac{1}{2} b \gamma-\frac{3}{2} \beta \partial_{z} c-\left(\partial_{z} \beta\right) c
\end{aligned}
$$

The expressions $\Phi_{I J}=\Phi_{I J 0}+\theta \Phi_{I J+}$, and $\Phi_{\beta}^{*}$ span a basis of superholomorphic $3 / 2$ differentials, while the $H_{\alpha}$ 's are super Beltrami differentials as all prescribed in [2], §3.3-§3.6. The dependence on $g$ is exhibited here to stress that the correlation functions are taken with respect to the complex structure of $g_{m n}$. 


\subsection{Deformation to $\hat{\Omega}_{I J}$ : the Chiral Measure}

It is important to keep in mind that in (2.8), both the finite-dimensional determinants and the correlation functions are expressed in terms of the metric $g_{m n}$, which depends in turn on the coordinates $\left(\hat{\Omega}_{I J}, \zeta^{\alpha}\right)$ as well as on the choice of Beltrami differential $\hat{\mu}$ going from $\hat{g}_{m n}$ to $g_{m n}$,

$$
\hat{\mu}_{\bar{w}}{ }^{w}=\frac{1}{2} \hat{g}_{w \bar{w}} g^{w w} \quad \Omega_{I J}-\hat{\Omega}_{I J}=i \int d^{2} w \hat{\mu}_{\bar{w}}{ }^{w} \omega_{I}(w) \omega_{J}(w)
$$

Here $w$ are holomorphic coordinates for $\hat{g}_{m n}$, so that $\hat{g}^{w w}=0$. The metric $g_{m n}$, and hence the Beltrami differential $\hat{\mu}$ is not unique. Different choices differ by a reparametrization of $\Sigma$, that is, infinitesimally by $\delta \hat{\mu}_{\bar{w}}^{w}=\partial_{\bar{w}} v^{w}$, where $v^{w}$ is a vector field.

In order to carry out the integration in the odd supermoduli $\zeta^{\alpha}$ with $\hat{\Omega}_{I J}$ as the remaining independent variables, it is essential to rewrite (2.8) in terms of $\hat{\Omega}_{I J}$. For the finite-dimensional determinants $\operatorname{det} \Phi_{I J+}\left(p_{a}\right)$ and $\operatorname{det}\left\langle H_{\alpha} \mid \Phi_{\beta}^{*}\right\rangle$, this is done in [2], §5.2 and $\S 6$. In correlation functions, the shift from background metric $g_{m n}$ to $\hat{g}_{m n}$ can be achieved by inserting in the correlators the term $\int \hat{\mu} T$, where the total stress tensor $T(z)$ is the sum of the matter and ghost stress tensors, $T(z)=T_{m}(z)+T_{g h}(z)$, with

$$
\begin{aligned}
T_{m}(z) & =-\frac{1}{2} \partial_{z} x_{+}^{\mu} \partial_{z} x_{+}^{\mu}(z)+\frac{1}{2} \psi_{+}^{\mu} \partial_{z} \psi_{+}^{\mu}(z) \\
T_{g h}(z) & =-\left(\partial_{z} b\right) c-2 b \partial_{z} c-\frac{3}{2} \beta \partial_{z} \gamma-\frac{1}{2}\left(\partial_{z} \beta\right) \gamma
\end{aligned}
$$

For the 0-point function, we obtain in this way the chiral measure derived in (1.9) of [2],

$$
\begin{gathered}
\mathcal{A}[\delta]=\frac{\left\langle\prod_{a=1}^{3} b\left(p_{a}\right) \prod_{\alpha=1}^{2} \delta\left(\beta\left(q_{\alpha}\right)\right)\right\rangle}{\operatorname{det} \Phi_{I J+}\left(p_{a}\right) \operatorname{det}\left\langle H_{\alpha} \mid \Phi_{\beta}^{*}\right\rangle}\left\{1-\frac{1}{8 \pi^{2}} \int d^{2} z \chi_{\bar{z}}{ }^{+} \int d^{2} w \chi_{\bar{w}}{ }^{+}\langle S(z) S(w)\rangle\right. \\
\left.+\frac{1}{2 \pi} \int d^{2} z \hat{\mu}_{\bar{z}}{ }^{z}\langle T(z)\rangle\right\}
\end{gathered}
$$

where all correlators are now taken with respect to the metric $\hat{g}_{m n}$.

\subsection{Deformation to $\hat{\Omega}_{I J}$ : the Vertex Operators}

For the $N$-point function, upon deformation to the metric $g_{m n}$ to the metric $\hat{g}_{m n}$ the chiral blocks (2.8) become,

$$
\mathcal{F}[\delta]=\frac{\left\langle\prod_{a} b\left(p_{a}\right) \prod_{\alpha} \delta\left(\beta\left(q_{\alpha}\right)\right)\right\rangle}{\operatorname{det} \Phi_{I J+}\left(p_{a}\right) \operatorname{det}\left\langle H_{\alpha} \mid \Phi_{\beta}^{*}\right\rangle}\left\langle\mathcal{Q}\left(p_{I}^{\mu}\right) \exp \left\{\frac{1}{2 \pi} \int(\chi S+\hat{\mu} T)\right\} \prod_{i=1}^{N} W\left(\mathbf{z}_{i} ; \epsilon_{i}, k_{i}\right)\right\rangle_{+}
$$

However, in the deformation to $\hat{g}_{m n}$, the volume form $d^{2 \mid 2} \mathbf{z}_{i} E\left(\mathbf{z}_{i}\right)$ has to be deformed also. Without this simultaneous deformation, the correlation functions would fail to be 
gauge-invariant. In particular, the amplitudes would end up depending on the choice of both the gravitino slice $\chi$ and the Beltrami differential $\hat{\mu}$. The deformation of the volume form $d^{2 \mid 2} \mathbf{z}_{i} E\left(\mathbf{z}_{i}\right)$ has to be carried out with some care, as it controls the complex type of the chiral vertex operators - viewed as differential forms on the worldsheet $\Sigma-$ and ultimately their transformation properties under changes of gauge slices.

In components, the volume form $E(\mathbf{z})$ for a supergeometry $\left(g_{m n}=e_{m}{ }^{a} e_{n}{ }^{b} \delta_{a b}, \chi\right)$ on the worldsheet $\Sigma$ is given by (see [7, eqs. (3.32)-(3.33))

$$
E=\operatorname{sdet} E_{M}^{A}=\operatorname{det}\left(e_{m}{ }^{a}\right)\left(1+\frac{1}{2} \theta \gamma^{n} \chi_{n}+\frac{1}{8} \theta \bar{\theta} \epsilon^{m n} \chi_{m} \gamma_{5} \chi_{n}\right)
$$

where $z$ is an isothermal coordinate with respect to the metric $g_{m n}$ and we have set auxiliary field to 0 . Under a deformation to the new complex structure $\hat{g}_{m n}$, we have

$$
\begin{aligned}
d \xi^{m} e_{m}{ }^{z} & =d z-\hat{\mu}_{\bar{z}}{ }^{z} d \bar{z} \\
\operatorname{det}\left(e_{m}{ }^{a}\right) & =1-\hat{\mu}_{z}^{\bar{z}} \hat{\mu}_{\bar{z}}{ }^{z}, \\
\theta \gamma^{n} \chi_{n} & =-\theta \hat{\mu}_{z}^{\bar{z}} \chi_{\bar{z}}{ }^{+}-\bar{\theta} \hat{\mu}_{\bar{z}}{ }^{z} \chi_{z}{ }^{-}
\end{aligned}
$$

where $z$ is now an isothermal coordinate with respect to $\hat{g}_{m n}$. Thus we obtain the key formula for the correct volume form for the vertex operators

$$
d^{2 \mid 2} \mathbf{z} E(\mathbf{z})=\left(d \bar{\theta} \wedge e^{\bar{z}}\right) \wedge\left(d \theta \wedge e^{z}\right)
$$

where we have set

$$
e^{z}=d z-\left(\hat{\mu}_{\bar{z}}^{z}+\frac{1}{2} \theta \chi_{\bar{z}}{ }^{+}\right) d \bar{z}, \quad \quad e^{\bar{z}}=d \bar{z}-\left(\hat{\mu}_{z}^{\bar{z}}+\frac{1}{2} \bar{\theta} \chi_{z}^{-}\right) d z
$$

We stress that this decomposition is chiral, in the sense that each factor $\left(d \theta \wedge e^{z}\right)$ or $\left(d \bar{\theta} \wedge e^{\bar{z}}\right)$ depends only on $\chi_{\bar{z}}{ }^{+}$or $\chi_{z}{ }^{-}$, but not on both. On the other hand, the decomposition is not holomorphic, in the sense that neither differential form $e^{z}$ or $e^{\bar{z}}$ is of pure $(1,0)$ or $(0,1)$ type.

This shows that the proper chiral vertex operator should incorporate the chiral volume form and be defined by

$$
\begin{aligned}
\mathcal{V}(z ; \epsilon, k) & =\int d \theta e^{z} W(\mathbf{z} ; \epsilon, k) \\
& =\int d \theta e^{z} \exp \left\{i k^{\mu}\left(x_{+}^{\mu}+\theta \psi_{+}\right)(z)+\epsilon^{\mu}\left(\psi_{+}^{\mu}+\theta \partial_{z} x_{+}^{\mu}\right)(z)\right\} \\
& =\epsilon^{\mu}\left\{\left(\partial_{z} x_{+}^{\mu}-i k^{\nu} \psi_{+}^{\mu} \psi_{+}^{\nu}\right)\left(d z-\hat{\mu}_{\bar{z}}^{z} d \bar{z}\right)-\frac{1}{2} d \bar{z}_{\bar{z}^{+}} \psi_{+}^{\mu}\right\} e^{i k \cdot x_{+}(z)}
\end{aligned}
$$

There is an overall sign issue since the ordering of the differentials and the polarization vector $\epsilon^{\mu}$ is a matter of convention. Throughout, the sign will be chosen as above. We note 
that the vertex operator $\mathcal{V}(z ; \epsilon, k)$ is now a 1 -form. As noted earlier for $e^{z}$, it is however a form with both $(1,0)$ and $(0,1)$ components.

It will be useful to view $\mathcal{V}$ as a sum of terms $\mathcal{V}^{(n)}$ which are of definite degree $n$ in the odd supermoduli $\zeta^{\alpha}$, as was done in (1.1), so that $\mathcal{V}=\mathcal{V}^{(0)}+\mathcal{V}^{(1)}+\mathcal{V}^{(2)}$. The components were given as in (1.2), and are recalled here for convenience,

$$
\begin{aligned}
\mathcal{V}^{(0)}(z ; \epsilon, k) & =\epsilon^{\mu} d z\left(\partial_{z} x_{+}^{\mu}-i k^{\nu} \psi_{+}^{\mu} \psi_{+}^{\nu}\right)(z) e^{i k \cdot x_{+}(z)} \\
\mathcal{V}^{(1)}(z ; \epsilon, k) & =-\frac{1}{2} \epsilon^{\mu} d \bar{z} \chi_{\bar{z}}^{+} \psi_{+}^{\mu}(z) e^{i k \cdot x_{+}(z)} \\
\mathcal{V}^{(2)}(z ; \epsilon, k) & =-\hat{\mu}_{\bar{z}}^{z} \frac{d \bar{z}}{d z} \mathcal{V}^{(0)}(z)
\end{aligned}
$$

The vertex $\mathcal{V}^{(0)}(z ; \epsilon, k)$ is of type $(1,0)$, while the vertices $\mathcal{V}^{(1)}(z ; \epsilon, k)$ and $\mathcal{V}^{(2)}(z ; \epsilon, k)$ are of type $(0,1)$. Finally, we stress that all quantities in the above expression have now been expressed in terms of the complex structure of the superperiod matrix $\hat{\Omega}_{I J}$. The original period matrix $\Omega_{I J}$ no longer appears explicitly in our considerations; henceforth, we simply denote $\hat{\Omega}_{I J}$ by $\Omega_{I J}$.

Altogether, we have obtained the following formula for the chirally symmetric contribution $\mathbf{A}[\delta]$ to the superstring measure for spin structure $\delta$

$$
\mathbf{A}[\delta]=\int_{\mathcal{M}_{2}} \int_{\Sigma^{N}} \int d p_{I}^{\mu}\left|\mathcal{B}[\delta]\left(z_{i} ; \epsilon_{i}, k_{i}, p_{I}^{\mu}\right)\right|^{2},
$$

where $\mathcal{B}[\delta]$ are the 1 -forms in each $z_{i}$ defined in (1.3).

\subsection{Non-Renormalization of the Super-Period Matrix}

The simple contraction, using (2.3), of the operator $Q\left(p_{I}\right)$, ignoring vertex operators and the insertions of the stress tensor and the supercurrent, gives rise to the following factor which is familiar from chiral splitting,

$$
\left\langle Q\left(p_{I}\right)\right\rangle=\exp \left\{i \pi p_{I}^{\mu} \hat{\Omega}_{I J} p_{J}^{\mu}\right\}
$$

Further contractions of $Q\left(p_{I}\right)$ with the stress tensor and supercurrent insertions produce corrections to this Gaussian. Actually, these corrections cancel one another, as is indeed expected to happen for consistency. The respective corrections are given as follows. For the insertion of the stress tensor, the contribution is given by,

$$
\begin{aligned}
\left\langle\frac{1}{2 \pi} \int \hat{\mu} T Q\left(p_{I}\right)\right\rangle_{(c)} & =\frac{1}{2}\left\langle Q\left(p_{I}\right)\right\rangle p_{I}^{\mu} p_{J}^{\mu} \oint_{B_{I}} d z \oint_{B_{J}} d w \int \frac{d^{2} u}{2 \pi} \hat{\mu}_{\bar{u}}{ }^{u} \partial_{z} \partial_{u} \ln E \partial_{z} \partial_{w} \ln E \\
& =-\pi p_{I}^{\mu} p_{J}^{\mu}\left\langle Q\left(p_{I}\right)\right\rangle \int \hat{\mu} \omega_{I} \omega_{J} \\
& =-i \pi p_{I}^{\mu} p_{J}^{\mu}\left\langle Q\left(p_{I}\right)\right\rangle\left(\hat{\Omega}_{I J}-\Omega_{I J}\right)
\end{aligned}
$$


where we have used the effective rule of (2.3) for the contraction of chiral bosons. Also, in passing from the second to the last line, we have used the definition of $\hat{\mu}$, in (2.10). For the insertion of two supercurrents, the contribution is given by

$$
\left\langle\frac{1}{2}\left(\frac{1}{2 \pi} \int \chi S\right)^{2} Q\left(p_{I}\right)\right\rangle_{(c)}
$$

Here, no contractions of both $x_{+}$and $\psi_{+}$are allowed between the two supercurrents, since such a term already belongs to the disconnected part. Performing first the $\psi_{+}$contraction, using (2.3) , we find the following expression,

$$
\left\langle\left(\frac{1}{2 \pi} \int \chi S\right)^{2}\right\rangle_{\psi_{+}}=-\frac{1}{4 \pi^{2}} \int d^{2} u \int d^{2} v \partial x_{+}^{\sigma}(u) \chi_{\bar{u}}{ }^{+} S_{\delta}(u, v) \chi_{\bar{v}}{ }^{+} \partial x_{+}^{\sigma}(v)
$$

Performing now also the contractions of $x_{+}$, using (2.3), we find

$$
\begin{aligned}
& \left\langle\frac{1}{2}\left(\frac{1}{2 \pi} \int \chi S\right)^{2} Q\left(p_{I}\right)\right\rangle_{(c)} \\
& \quad=-\frac{p_{I}^{\mu} p_{J}^{\mu}}{32 \pi^{2}}\left\langle Q\left(p_{I}\right)\right\rangle \oint_{B_{I}} d z \oint_{B_{J}} d w \int d^{2} u \int d^{2} v \chi_{\bar{u}}{ }^{+} S_{\delta}(u, v) \chi_{\bar{v}}{ }^{+} \partial_{z} \partial_{u} \ln E \partial_{w} \partial_{v} \ln E \\
& \quad=\frac{1}{8} p_{I}^{\mu} p_{J}^{\mu}\left\langle Q\left(p_{I}\right)\right\rangle \int d^{2} u \int d^{2} v \omega_{I}(u) \chi_{\bar{u}}{ }^{+} S_{\delta}(u, v) \chi_{\bar{v}}{ }^{+} \omega_{J}(v) \\
& \quad=\pi i p_{I}^{\mu} p_{J}^{\mu}\left\langle Q\left(p_{I}\right)\right\rangle\left(\hat{\Omega}_{I J}-\Omega_{I J}\right)
\end{aligned}
$$

In the passage to the last line, we have used the definition of $\hat{\Omega}_{I J}$ in (1.7). Clearly, the contributions from the insertion of $T$ in (2.22) and of $S S$ in (2.25) cancel one another. 


\section{The $d x_{+}^{\mu}$ Formulation of Vertex Operators}

The conceptual difficulties associated with the fact that the full vertex operator $\mathcal{V}$ is now gauge-slice dependent have been stressed in the Introduction, section $\S 1$. The gauge-slice dependent corrections $\mathcal{V}^{(1)}$ and $\mathcal{V}^{(2)}$ give rise to some practical difficulties as well. In practice, it is often convenient to choose Dirac point masses for the gravitino slice $\chi_{\bar{z}}{ }^{+}$and the Beltrami differential $\hat{\mu}_{\bar{z}}{ }^{z}$. How to do so when $\chi_{\bar{z}}{ }^{+}$and $\hat{\mu}_{\bar{z}}{ }^{z}$ appear in an "unintegrated" form in a chiral amplitude, as in $\mathcal{V}^{(1)}$ and $\mathcal{V}^{(2)}$, is fraught with difficulties, and can easily lead to contradicting outcomes.

In this section, we show that there is an alternate formulation of the full vertex operators $\mathcal{V}$, where the unintegrated $\chi_{\bar{z}}{ }^{+}$cancel out. This shows that, when evaluating the $N$-point function, it is safe to take $\chi_{\bar{z}}{ }^{+}$to consist of Dirac point masses at the outset. However, the Beltrami differential $\hat{\mu}_{\bar{z}}{ }^{z}$ still appears in an unintegrated form, and it is not reliable to take it as consisting of Dirac point masses. These basic guidelines will be followed in the explicit evaluation of the $N$-point function for $N \leq 4$ in 8 .

The key to the alternative formulation is a natural geometric object $\mathcal{U}(z)$, defined by

$$
\mathcal{U}(z ; \epsilon, k) \equiv \epsilon^{\mu}\left(d x_{+}^{\mu}-i k^{\nu} d z \psi_{+}^{\mu} \psi_{+}^{\nu}\right)(z) e^{i k \cdot x_{+}(z)}
$$

Here, $d$ is the differential operator acting on the point of the vertex $z$. This vertex differs from $\mathcal{V}^{(0)}$ as follows,

$$
\begin{aligned}
& \mathcal{U}(z ; \epsilon, k)=\mathcal{V}^{(0)}(z ; \epsilon, k)+\mathcal{K}(z ; \epsilon, k) \\
& \mathcal{K}(z ; \epsilon, k)=\epsilon^{\mu} d \bar{z} \partial_{\bar{z}} x_{+}^{\mu}(z) e^{i k \cdot x_{+}(z)}
\end{aligned}
$$

We now wish to re-express the amplitude in terms of the vertices $\mathcal{U}$. The difference between the vertices $\mathcal{V}^{(0)}\left(z_{i} ; \epsilon_{i}, k_{i}\right)$ and $\mathcal{U}\left(z_{i} ; \epsilon_{i}, k_{i}\right)$ involves a $\partial_{\bar{z}} x_{+}\left(z_{i}\right)$ factor which, upon contraction of the field $x_{+}$, produces contributions at the points where $x_{+}$is not complex analytic. There are four instances when non-analyticities appear,

1. at insertion points $z_{j}$, different from $z_{i}$;

2. at the insertion points of the supercurrent $S$;

3. at the insertion of the stress tensor $T$;

4. at the insertion of the internal momentum operator in $Q\left(p_{I}\right)$.

The effects of 1 are immaterial in view of the "cancelled propagator argument", i.e. the analyticity as a function of external momenta. 


\subsection{Preliminaries}

Thus, we need to obtain only the contributions from 2,3 , and 4 . These are computed using the following contraction rules, where only the factor $\partial_{\bar{z}} x_{+}^{\mu}(z)$ is contracted but all other fields are left for contraction at a later stage. We denote this procedure by $\langle\cdots\rangle_{*}$. We have,

$$
\begin{aligned}
\left\langle\frac{1}{2 \pi} \int \chi S \partial_{\bar{z}} x_{+}^{\mu}(z)\right\rangle_{*} & =-\frac{1}{2} \chi_{\bar{z}}{ }^{+} \psi_{+}^{\mu}(z) \\
\left\langle\frac{1}{2 \pi} \int \hat{\mu} T \partial_{\bar{z}} x_{+}^{\mu}(z)\right\rangle_{*} & =-\hat{\mu}_{\bar{z}}{ }^{z} \partial_{z} x_{+}^{\mu}(z) \\
\left\langle Q\left(p_{I}\right) \partial_{\bar{z}} x_{+}^{\mu}(z)\right\rangle_{*} & =Q\left(p_{I}\right) i p_{I}^{\mu} \partial_{\bar{z}} \oint_{B_{I}} d w\left\langle\partial_{w} x_{+}(w) x_{+}(z)\right\rangle=0
\end{aligned}
$$

From the last line, it follows that contractions of item 4 above do not occur. It is useful to have the contraction of the entire operator $\mathcal{K}$, using the same rules and the same notations. After a brief calculation, using (3.3), we find $\left\langle Q\left(p_{I}\right) \mathcal{K}_{i}\right\rangle_{*}=0$ and

$$
\begin{aligned}
\left\langle\frac{1}{2 \pi} \int \chi S \mathcal{K}_{i}\right\rangle_{*} & =\mathcal{V}_{i}^{(1)} \\
\left\langle\frac{1}{2 \pi} \int \hat{\mu} T \mathcal{K}_{i}\right\rangle_{*} & =\mathcal{V}_{i}^{(2)}-\mathrm{E}_{i}
\end{aligned}
$$

where $\mathrm{E}_{i}$ is given by

$$
\mathrm{E}_{i}=i \hat{\mu}_{\bar{z}_{i}}{ }^{z_{i}} d \bar{z}_{i} \epsilon_{i}^{\mu} k_{i}^{\nu} \psi_{+}^{\mu} \psi_{+}^{\nu}\left(z_{i}\right) e^{i k_{i} \cdot x_{+}\left(z_{i}\right)}
$$

This contribution has a natural interpretation. The product $\psi_{+}^{\mu} \psi_{+}^{\nu}$ is the $z$-component of a local Lorentz vector, so that the Lorentz invariant $(d z-\hat{\mu} d \bar{z}) \psi_{+}^{\mu} \psi_{+}^{\nu}$ is actually the proper 1 -form.

\subsection{Reformulation}

We reformulate $\mathcal{Y}_{1}, \mathcal{Y}_{2}, \mathcal{Y}_{3}, \mathcal{Y}_{4}, \mathcal{Y}_{5}$ in terms of the vertices $\mathcal{U}_{i}$. Substitution yields,

$$
\begin{aligned}
\mathcal{Y}_{1}= & \frac{1}{8 \pi^{2}}\left\langle Q\left(p_{I}\right) \int \chi S \int \chi S \prod_{i=1}^{N} \mathcal{U}_{i}\right\rangle_{(c)}-\sum_{i=1}^{N} \frac{1}{8 \pi^{2}}\left\langle Q\left(p_{I}\right) \int \chi S \int \chi S \mathcal{K}_{i} \prod_{j \neq i}^{N} \mathcal{U}_{j}\right\rangle_{(c)} \\
& +\frac{1}{2} \sum_{i \neq j}^{N} \frac{1}{8 \pi^{2}}\left\langle Q\left(p_{I}\right) \int \chi S \int \chi S \mathcal{K}_{i} \mathcal{K}_{j} \prod_{l \neq i, j}^{N} \mathcal{U}_{l}\right\rangle_{(c)}
\end{aligned}
$$

Using the $\langle\cdots\rangle_{*}$ contractions, we obtain,

$$
\mathcal{Y}_{1}=\frac{1}{8 \pi^{2}}\left\langle Q\left(p_{I}\right) \int \chi S \int \chi S \prod_{i=1}^{N} \mathcal{U}_{i}\right\rangle_{(c)}-\sum_{i=1}^{N} \frac{1}{2 \pi}\left\langle Q\left(p_{I}\right) \int \chi S \mathcal{V}_{i}^{(1)} \prod_{j \neq i}^{N} \mathcal{U}_{j}\right\rangle
$$




$$
+\frac{1}{2} \sum_{i \neq j}^{N}\left\langle Q\left(p_{I}\right) \mathcal{V}_{i}^{(1)} \mathcal{V}_{j}^{(1)} \prod_{l \neq i, j}^{N} \mathcal{U}_{l}\right\rangle
$$

The remaining contributions are calculated in a similar manner and are given by

$$
\begin{aligned}
& \mathcal{Y}_{2}=\frac{1}{2 \pi}\left\langle Q\left(p_{I}\right) \int \hat{\mu} T \prod_{i=1}^{N} \mathcal{U}_{i}\right\rangle_{(c)}+\sum_{i=1}^{N}\left\langle Q\left(p_{I}\right)\left(-\mathcal{V}_{i}^{(2)}+\mathrm{七}_{i}\right) \prod_{j \neq i}^{N} \mathcal{U}_{j}\right\rangle \\
& \mathcal{Y}_{3}=\frac{1}{2 \pi} \sum_{i=1}^{N}\left\langle Q\left(p_{I}\right) \int \chi S \mathcal{V}_{i}^{(1)} \prod_{j \neq i}^{N} \mathcal{U}_{j}\right\rangle-\sum_{i \neq j j}^{N}\left\langle Q\left(p_{I}\right) \mathcal{V}_{i}^{(1)} \mathcal{V}_{j}^{(1)} \prod_{l \neq i, j}^{N} \mathcal{U}_{l}\right\rangle \\
& \mathcal{Y}_{4}=\frac{1}{2} \sum_{i \neq j}^{N}\left\langle Q\left(p_{I}\right) \mathcal{V}_{i}^{(1)} \mathcal{V}_{j}^{(1)} \prod_{l \neq i, j}^{N} \mathcal{U}_{l}\right\rangle \\
& \mathcal{Y}_{5}=\sum_{i=1}^{N}\left\langle Q\left(p_{I}\right) \mathcal{V}_{i}^{(2)} \prod_{j \neq i}^{N} \mathcal{U}_{j}\right\rangle
\end{aligned}
$$

Putting all together, we obtain our final result,

$$
\begin{aligned}
\sum_{a=1}^{5} \mathcal{Y}_{a}= & \frac{1}{8 \pi^{2}}\left\langle Q\left(p_{I}\right) \int \chi S \int \chi S \prod_{i=1}^{N} \mathcal{U}_{i}\right\rangle_{(c)} \\
& +\frac{1}{2 \pi}\left\langle Q\left(p_{I}\right) \int \hat{\mu} T \prod_{i=1}^{N} \mathcal{U}_{i}\right\rangle_{(c)}+\sum_{i=1}^{N}\left\langle Q\left(p_{I}\right) \mathrm{E}_{i} \prod_{j \neq i}^{N}\right\rangle
\end{aligned}
$$

Clearly, in terms of the vertex $\mathcal{U}$, the amplitude simplifies considerably. 


\section{The Amplitudes $\mathcal{B}[\delta]$ as Closed Differential Forms}

The first important property of the vertex operators $\mathcal{V}_{i}$ is that their correlation functions are closed differential forms with respect to each $z_{i}$. This property is manifest for the disconnected part $\mathcal{B}[\delta]^{(d)}$ since the correlator $\left\langle Q\left(p_{I}\right) \prod_{i} \mathcal{V}^{(0)}\right\rangle$ is a holomorphic 1-form in each $z_{i}$ away from coincident points. For the connected part, the closedness is equivalent to the equation,

$$
d_{i} \sum_{a=1}^{5} \mathcal{Y}_{a}=0, \quad 1 \leq i \leq N .
$$

There are at least two ways of seeing this. The first way makes use of the superholomorphicity of the chiral blocks. The second way relies on the representation (3.9) of the $\mathcal{Y}_{a}$ in terms of the vertices $\mathcal{U}_{i}$ obtained in $§ 2.6$. We discuss them both.

In the first way, we observe that the closedness property of a differential form is a notion which depends only on the $C^{\infty}$ structure, and not on the complex structure. Thus we can verify it in the original conformal coordinates $z$ of the metric $g_{m n}$, and do not need to deform to $\hat{\Omega}_{I J}$ with the Beltrami differential $\hat{\mu}_{\bar{z}}{ }^{z}$. The chiral volume form is then $d \theta \wedge e^{z}=d \theta \wedge\left(d z-\frac{1}{2} \theta \chi_{\bar{z}}{ }^{+} d \bar{z}\right)$, and it suffices to show that the expression

$$
\int d \theta \wedge\left(d z-\frac{1}{2} \theta \chi_{\bar{z}}{ }^{+} d \bar{z}\right) \mathcal{F}[\delta]\left(z, \theta, \mathbf{z}_{2}, \cdots, \mathbf{z}_{N}\right)=d z \mathcal{F}_{+}[\delta]-\frac{1}{2} d \bar{z} \chi_{\bar{z}}{ }^{+} \mathcal{F}_{0}[\delta]
$$

is a closed 1-form in $z$. Here we have set $\left(z_{1}, \theta_{1}\right)=(z, \theta), \mathcal{F}[\delta]=\mathcal{F}_{0}[\delta]+\theta \mathcal{F}_{+}[\delta]$ to simplify notation, the other variables $\mathbf{z}_{i}$ being treated successively in the same way. However, the chiral blocks $\mathcal{F}[\delta]$ are superholomorphic, that is, The chiral blocks $\mathcal{F}[\delta]$ are superholomorphic, i.e.,

$$
\mathcal{D}_{-} \mathcal{F}[\delta]=0, \quad \mathbf{z}_{\mathbf{i}} \neq \mathbf{z}_{j}
$$

Decomposing this superholomorphicity property into components, we have

$$
\mathcal{D}_{-} \mathcal{F}[\delta]=\bar{\theta}\left(\partial_{\bar{z}} \mathcal{F}_{+}[\delta]+\frac{1}{2} \chi_{\bar{z}}+\mathcal{F}_{+}[\delta]\right)+\bar{\theta} \theta\left(\partial_{\bar{z}} \mathcal{F}_{+}[\delta]+\frac{1}{2} \partial_{z}\left(\chi_{\bar{z}}+\mathcal{F}_{0}\right)[\delta]\right)
$$

Thus, the holomorphicity condition $\mathcal{D}_{-} \mathcal{F}[\delta]=0$ implies that $\partial_{\bar{z}} \mathcal{F}_{+}[\delta]+\frac{1}{2} \partial_{z}\left(\chi_{\bar{z}}{ }^{+} \mathcal{F}_{0}[\delta]\right)=0$, which is exactly the condition that the 1-form (4.2) be closed.

The second way is based on the conformal coordinates $z$ for the metric $\hat{g}_{m n}$. It is slightly longer, but it illustrates well the roles of the various terms in the representation (3.9). The exterior derivative of the amplitudes with respect to any one of the vertex insertion points is computed from the exterior derivative of the individual vertex operators, viewed as differential forms. The differential of the individual vertex is easily obtained,

$$
d \mathcal{U}=\epsilon^{\mu} k^{\nu}\left\{-i d x_{+}^{\mu} \wedge d x_{+}^{\nu}+k^{\sigma} d x_{+}^{\sigma} \wedge d z \psi_{+}^{\mu} \psi_{+}^{\nu}-i d\left(d z \psi_{+}^{\mu} \psi_{+}^{\nu}\right)\right\} e^{i k \cdot x_{+}}
$$


We begin by focussing on the $\partial_{\bar{z}}$ parts in $d \mathcal{U}$, and their contractions inside the amplitude. By the "cancelled propagator" argument, any contractions of the $\partial_{\bar{z}}$ terms with fields in other vertex operators will yield $\delta\left(z_{i}, z_{j}\right)$ contact terms which, by external momentum analyticity, must vanish. Thus, the $\partial_{\bar{z}}$ parts of $d \mathcal{U}$ must be contracted only with the operators $S$ and $T$. Preliminary formulas that will come in handy are as follows,

$$
\begin{aligned}
\left\langle\frac{1}{2 \pi} \int \hat{\mu} T \partial_{\bar{z}} x_{+}^{\mu}(z)\right\rangle_{*} & =-\hat{\mu}_{\bar{z}}{ }^{z} \partial_{z} x_{+}^{\mu}(z) \\
\left\langle\frac{1}{2 \pi} \int \hat{\mu} T \partial_{\bar{z}}\left(\psi_{+}^{\mu} \psi_{+}^{\nu}\right)\right\rangle_{*} & =-\partial_{z}\left(\hat{\mu}_{\bar{z}}^{z} \psi_{+}^{\mu} \psi_{+}^{\nu}\right)(z)
\end{aligned}
$$

which may be derived using the standard Green functions for $x_{+}$and $\psi_{+}$. These expressions are intimately related with the corresponding diffeomorphism transformations of these fields (see e.g. [7], §III.C).

\section{$4.1 \hat{\mu}$-Dependence}

The first contraction we need to evaluate is

$$
\left\langle\frac{1}{2 \pi} \int \hat{\mu} T d \mathcal{U}(z)\right\rangle_{*}
$$

Using (4.6), the first term of (4.5) contracts as follows,

$$
\left\langle\frac{1}{2 \pi} \int \hat{\mu} T\left(\partial_{z} x_{+}^{\mu} \partial_{\bar{z}} x_{+}^{\nu}-\partial_{z} x_{+}^{\nu} \partial_{\bar{z}} x_{+}^{\mu}\right)\right\rangle_{*}=-\hat{\mu}_{\bar{z}}^{z} \partial_{z} x_{+}^{[\mu} \partial_{z} x_{+}^{\nu]}=0
$$

and therefore does not contribute to (4.7). The second and third terms in (4.5) contract as follows,

$$
\begin{aligned}
\left\langle\frac{1}{2 \pi} \int \hat{\mu} T \partial_{\bar{z}} x_{+}^{\sigma} \psi_{+}^{\mu} \psi_{+}^{\nu} e^{i k \cdot x_{+}}\right\rangle_{*} & =-\hat{\mu}_{\bar{z}}^{z} \partial_{z} x_{+}^{\sigma} \psi_{+}^{\mu} \psi_{+}^{\nu} e^{i k \cdot x_{+}} \\
\left\langle\frac{1}{2 \pi} \int \hat{\mu} T \partial_{\bar{z}}\left(\psi_{+}^{\mu} \psi_{+}^{\nu}\right) e^{i k \cdot x_{+}}\right\rangle_{*} & =-\partial_{z}\left(\hat{\mu}_{\bar{z}}^{z} \psi_{+}^{\mu} \psi_{+}^{\nu}(z)\right) e^{i k \cdot x_{+}}
\end{aligned}
$$

Combining both of these, we get

$$
\left\langle\frac{1}{2 \pi} \int \hat{\mu} T\left[i k^{\sigma} \partial_{\bar{z}} x_{+}^{\sigma} \psi_{+}^{\mu} \psi_{+}^{\nu}+\partial_{\bar{z}}\left(\psi_{+}^{\mu} \psi_{+}^{\nu}\right)\right] e^{i k \cdot x_{+}}\right\rangle_{*}=-\partial_{z}\left(\hat{\mu}_{\bar{z}}^{z} \psi_{+}^{\mu} \psi_{+}^{\nu} e^{i k \cdot x_{+}}\right)
$$

and further combining with the momenta and polarization vector, we obtain,

$$
\left\langle\frac{1}{2 \pi} \int \hat{\mu} T d_{i} \mathcal{U}_{i}\left(z_{i}\right)\right\rangle_{*}=-i \epsilon_{i}^{\mu} k_{i}^{\nu} d z_{i} \wedge d \bar{z}_{i} \partial_{z_{i}}\left(\hat{\mu}_{\bar{z}_{i}} z_{i} \psi_{+}^{\mu} \psi_{+}^{\nu}\left(z_{i}\right) e^{i k \cdot x_{+}}\right)
$$


Recalling now that there is also an additional contribution to the amplitude denoted by $\mathrm{Ł}_{i}$, which produces the following derivative term,

$$
d_{i} \mathrm{E}_{i}=i \epsilon_{i}^{\mu} k_{i}^{\nu} d z_{i} \wedge d \bar{z}_{i} \partial_{z_{i}}\left(\hat{\mu}_{\bar{z}_{i}} z_{i} \psi_{+}^{\mu} \psi_{+}^{\nu}\left(z_{i}\right) e^{i k_{i} \cdot x_{+}\left(z_{i}\right)}\right)
$$

we see that the sum of all contributions involving $\hat{\mu}$ cancel as follows,

$$
\left\langle\frac{1}{2 \pi} \int \hat{\mu} T d_{i} \mathcal{U}_{i}\left(z_{i}\right)\right\rangle_{*}+d_{i} \mathrm{E}_{i}=0
$$

Hence, all contractions of the $\partial_{\bar{z}}$ terms with the stress tensor vanish.

\section{$4.2 \chi$-Dependence}

The $\chi$-dependence governs the contractions of the $\partial_{\bar{z}}$-terms with the supercurrent $S$, and we make use of the following preliminary formulas,

$$
\begin{aligned}
\left\langle\frac{1}{2 \pi} \int \chi S \partial_{\bar{z}} x_{+}^{\mu}(z)\right\rangle_{*} & =-\frac{1}{2} \chi_{\bar{z}}{ }^{+} \psi_{+}^{\mu}(z) \\
\left\langle\frac{1}{2 \pi} \int \chi S \partial_{\bar{z}}\left(\psi_{+}^{\mu} \psi_{+}^{\nu}\right)\right\rangle_{*} & =-\frac{1}{2} \chi_{\bar{z}}{ }^{+}\left(\partial_{z} x_{+}^{\mu} \psi_{+}^{\nu}-\partial_{z} x_{+}^{\nu} \psi_{+}^{\mu}\right)(z)
\end{aligned}
$$

which are intimately connected with local worldsheet supersymmetry transformations. We need to evaluate the following contractions,

$$
\left\langle\frac{1}{2 \pi} \int \chi S d \mathcal{U}(z)\right\rangle_{*}
$$

The three parts of $d \mathcal{U}$ given rise to the following contractions,

$$
\begin{aligned}
\left\langle\frac{1}{2 \pi} \int \chi S\left(\partial_{z} x_{+}^{\mu} \partial_{\bar{z}} x_{+}^{\nu}-\partial_{z} x_{+}^{\nu} \partial_{\bar{z}} x_{+}^{\mu}\right) e^{i k \cdot x_{+}}\right\rangle_{*} & =-\frac{1}{2} \chi_{\bar{z}}{ }^{+}\left(\partial_{z} x_{+}^{\mu} \psi_{+}^{\nu}-\partial_{z} x_{+}^{\nu} \psi_{+}^{\mu}\right) e^{i k \cdot x_{+}} \\
\left\langle\frac{1}{2 \pi} \int \chi S \partial_{\bar{z}} x_{+}^{\sigma} \psi_{+}^{\mu} \psi_{+}^{\nu} e^{i k \cdot x_{+}}\right\rangle_{*} & =-\frac{1}{2} \chi_{\bar{z}}{ }^{+} \psi_{+}^{\sigma} \psi_{+}^{\mu} \psi_{+}^{\nu} e^{i k \cdot x_{+}} \\
\left\langle\frac{1}{2 \pi} \int \chi S \partial_{\bar{z}}\left(\psi_{+}^{\mu} \psi_{+}^{\nu}\right) e^{i k \cdot x_{+}}\right\rangle_{*} & =-\frac{1}{2} \chi_{\bar{z}}{ }^{+}\left(\partial_{z} x_{+}^{\mu} \psi_{+}^{\nu}-\partial_{z} x_{+}^{\nu} \psi_{+}^{\mu}\right) e^{i k \cdot x_{+}}
\end{aligned}
$$

Inserting the factors of momenta and polarization vectors in the second line gives,

$$
\left\langle\frac{1}{2 \pi} \int \chi S \epsilon^{\mu} k^{\nu} k^{\sigma} \partial_{\bar{z}} x_{+}^{\sigma} \psi_{+}^{\mu} \psi_{+}^{\nu} e^{i k \cdot x_{+}}\right\rangle_{*}=-\frac{1}{2} \epsilon^{\mu} \chi_{\bar{z}}{ }^{+}\left(k \cdot \psi_{+}\right) \psi_{+}^{\mu}\left(k \cdot \psi_{+}\right) e^{i k \cdot x_{+}}=0
$$

The cancellation occurs because of the square of the single fermionic field factor vanishes, $\left(k \cdot \psi_{+}\right)\left(k \cdot \psi_{+}\right)=0$. Using the cancellation generated by the second line, the first and third lines combine to yield 0 to the correlator of $d \mathcal{U}$, so that we have,

$$
\left\langle\frac{1}{2 \pi} \int \chi S d_{i} \mathcal{U}_{i}\left(z_{i}\right)\right\rangle_{*}=0
$$

Therefore, all contractions of all $\partial_{\bar{z}}$ terms in $d \mathcal{U}$ with the supercurrent vanish as well. The closedness of the differential form $\sum_{a=1}^{5} \mathcal{Y}_{a}$ is established. 


\section{Gauge Slice Variations as Exact Differentials}

In this section, we prove that the full chiral amplitudes, including the chiral contribution of the volume form, transform in a simple manner under changes in slice for the Beltrami differentials $\hat{\mu}$ and $\chi$. The changes of the chiral amplitudes are by the addition of an exact differential of a well-defined and single-valued differential in the external insertion points.

\subsection{Dependence on the $\hat{\mu}$-Slice}

Dependence on the $\hat{\mu}$-slice is investigated using a variation of the slice according to the following rule, which leave $\hat{\Omega}_{I J}$ invariant

$$
\begin{aligned}
\delta_{v} \hat{\mu}_{\bar{z}}^{z}(z) & =\partial_{\bar{z}} v^{z}(z) \\
\delta_{v} \chi_{\bar{z}}{ }^{+}(z) & =0
\end{aligned}
$$

Here, $v^{z}$ is a single-valued, non-singular vector field. Also, since $\hat{\mu}$ is bilinear in the odd supermoduli $\zeta^{\alpha}, v^{z}$ must be bilinear in $\zeta^{\alpha}$.

The variation of $\hat{\mu}$ affects the amplitude $\mathcal{B}[\delta]$ as follows. The variation of the disconnected part, $\mathcal{B}[\delta]^{(d)}$ has already been carried out in [2] and cancels. The variation of the connected part may be computed from the variations of the terms in $\mathcal{B}[\delta]^{(c)}$. To determine the latter, we need the variations of the vertex operators, which are given by

$$
\begin{aligned}
& \delta_{v} \mathcal{V}^{(0)}(z)=0 \\
& \delta_{v} \mathcal{V}^{(1)}(z)=0 \\
& \delta_{v} \mathcal{V}^{(2)}(z)=-\partial_{\bar{z}} v^{z} \frac{d \bar{z}}{d z} \mathcal{V}^{(0)}(z)
\end{aligned}
$$

As a result, we have $\delta_{v} \mathcal{Y}_{1}=\delta_{v} \mathcal{Y}_{3}=\delta_{v} \mathcal{Y}_{4}=0$. Notice that we also have $\delta_{v}\left(d \mu_{0}[\delta]\right)=0$, in view of the results of paper II. The variations that remain are as follows,

$$
\begin{aligned}
& \delta_{v} \mathcal{Y}_{2}=\frac{1}{2 \pi} \int d^{2} z \partial_{\bar{z}} v^{z}\left\langle Q\left(p_{I}\right) T(z) \prod_{i=1}^{N} \mathcal{V}_{i}^{(0)}\right\rangle_{(c)} \\
& \delta_{v} \mathcal{Y}_{5}=-\sum_{i=1}^{N} \partial_{\bar{z}_{i}} v^{z_{i}}\left(z_{i}\right) \frac{d \bar{z}_{i}}{d z_{i}}\left\langle Q\left(p_{I}\right) \prod_{j=1}^{N} \mathcal{V}_{j}^{(0)}\right\rangle
\end{aligned}
$$

The first variation may be computed using the following standard OPEs,

$$
\begin{aligned}
T(z) \partial_{w} x_{+}(w) & =\frac{1}{(z-w)^{2}} \partial_{w} x_{+}(w)+\frac{1}{z-w} \partial_{w}^{2} x_{+}(w)+\emptyset(1) \\
T(z) \psi_{+}^{\mu} \psi_{+}^{\nu}(w) & =\frac{1}{(z-w)^{2}} \psi_{+}^{\mu} \psi_{+}^{\nu}(w)+\frac{1}{z-w} \partial_{w}\left(\psi_{+}^{\mu} \psi_{+}^{\nu}\right)(w)+\emptyset(1)
\end{aligned}
$$


and hence

$$
\begin{aligned}
\frac{1}{2 \pi} \int d^{2} z \partial_{\bar{z}} v^{z} T(z) \partial_{w} x_{+}(w) & =-\partial_{w}\left(v^{w} \partial_{w} x_{+}(w)\right) \\
\frac{1}{2 \pi} \int d^{2} z \partial_{\bar{z}} v^{z} T(z) \psi_{+}^{\mu} \psi_{+}^{\nu}(w) & =-\partial_{w}\left(v^{w} \psi_{+}^{\mu} \psi_{+}^{\nu}(w)\right)
\end{aligned}
$$

From these relations, we deduce the following useful identities,

$$
\begin{aligned}
\frac{1}{2 \pi} \int d^{2} z \partial_{\bar{z}} v^{z} T(z) Q\left(p_{I}\right) & =-p_{I}^{\mu} \oint_{B_{I}} d w \partial_{w}\left(v^{w} \partial_{w} x_{+}(w)\right) Q\left(p_{I}\right)=0 \\
\frac{1}{2 \pi} \int d^{2} z \partial_{\bar{z}} v^{z} T(z) \mathcal{V}^{(0)}(w) & =-\partial_{w}\left(v^{w} \mathcal{V}^{(0)}(w)\right)
\end{aligned}
$$

Applying these results to the calculation of $\mathcal{Y}_{2}$, we find,

$$
\delta_{v} \mathcal{Y}_{2}=-\sum_{i=1}^{N} \partial_{z_{i}}\left(v^{z_{i}}\left\langle Q\left(p_{I}\right) \prod_{j=1}^{N} \mathcal{V}_{j}^{(0)}\right\rangle\right)
$$

The second variation may be recast as follows,

$$
\begin{aligned}
\delta_{v} \mathcal{Y}_{5}= & -\sum_{i=1}^{N} \frac{d \bar{z}_{i}}{d z_{i}} \partial_{\bar{z}_{i}}\left(v^{z_{i}}\left(z_{i}\right)\left\langle Q\left(p_{I}\right) \prod_{j=1}^{N} \mathcal{V}_{j}^{(0)}\right\rangle\right) \\
& +\sum_{i=1}^{N} \frac{d \bar{z}_{i}}{d z_{i}} v^{z_{i}}\left(z_{i}\right) \partial_{\bar{z}_{i}}\left\langle Q\left(p_{I}\right) \prod_{j=1}^{N} \mathcal{V}_{j}^{(0)}\right\rangle
\end{aligned}
$$

As it is assumed throughout that the points $z_{i}$ are distinct, i.e. $z_{i} \neq z_{j}$ whenever $i \neq j$, the second term above vanishes since away from the coincident locus, these correlators are holomorphic in $z_{i}$. Combining both variations, and recognizing the components of the exact differential $d_{i} \equiv d z_{i} \partial_{z_{i}}+d \bar{z}_{i} \partial_{\bar{z}_{i}}$, we have,

$$
\delta_{v} \mathcal{B}[\delta]^{(c)}=d \mu_{0}[\delta]\left(\delta_{v} \mathcal{Y}_{2}+\delta_{v} \mathcal{Y}_{5}\right)=\sum_{i=1}^{N} d_{i} \mathcal{R}_{i}^{v}[\delta]
$$

Here, the differential forms $\mathcal{R}_{i}^{v}[\delta]$ are given by

$$
\mathcal{R}_{i}^{v}[\delta]=-d \mu_{0}[\delta] v^{z_{i}}\left(d z_{i}\right)^{-1}\left\langle Q\left(p_{I}\right) \prod_{j=1}^{N} \mathcal{V}_{j}^{(0)}\right\rangle
$$

This form has the following properties,

- $\mathcal{R}_{i}^{v}[\delta]$ is a form of type $(1,0)$ in $z_{j}$ for $j \neq i$;

- $\mathcal{R}_{i}^{v}[\delta]$ is a form of type $(0,0)$ in $z_{i}$;

- $\mathcal{R}_{i}^{v}[\delta]$ is holomorphic in $z_{j}$ for $j \neq i$.

This concludes the proof of our assertion on $\hat{\mu}$-slice dependence. 


\subsection{Dependence on the $\chi$-Slice}

The $\chi$-slice variation is given by the following rule,

$$
\begin{aligned}
\delta_{\xi} \mu_{\bar{z}}{ }^{z}(z) & =\xi^{+} \chi_{\bar{z}}{ }^{+}(z) \\
\delta_{\xi} \chi_{\bar{z}}{ }^{+}(z) & =-2 \partial_{\bar{z}} \xi^{+}(z)
\end{aligned}
$$

Since $\chi$ itself is linear in the odd supermoduli $\zeta^{\alpha}, \xi^{+}$is also linear in $\zeta^{\alpha}$. Furthermore, $\xi^{+}$ is a single-valued, non-singular spinor field.

The variation of $\chi$ affects the amplitude as follows. The variation of the disconnected part, $\mathcal{B}[\delta]^{(d)}$, has already been carried out in [2] and cancels. The variation of the connected part may be computed from the variations of the terms in $\mathcal{B}[\delta]^{(c)}$. (Notice that we have $\delta_{\xi}\left(d \mu_{0}[\delta]\right)=0$ automatically.) To determine these, we need the variations of the vertex operator pieces, which are given by,

$$
\begin{aligned}
\delta_{\xi} \mathcal{V}^{(0)}(z) & =0 \\
\delta_{\xi} \mathcal{V}^{(1)}(z) & =-d \bar{z}\left(\partial_{\bar{z}} \xi^{+}(z)\right) \epsilon^{\mu} \psi_{+}^{\mu}(z) e^{i k \cdot x_{+}(z)} \\
\delta_{\xi} \mathcal{V}^{(2)}(z) & =-\xi^{+} \chi_{\bar{z}}{ }^{+} \frac{d \bar{z}}{d z} \mathcal{V}^{(0)}(z)
\end{aligned}
$$

Using these ingredients, we can recast the variations of the $\mathcal{Y}$ 's in a more explicit form,

$$
\begin{aligned}
& \delta_{\xi} \mathcal{Y}_{1}=\frac{1}{4 \pi^{2}}\left\langle Q\left(p_{I}\right) \int d^{2} z \delta_{\xi} \chi_{\bar{z}}^{+}(z) S(z) \int \chi S \prod_{i=1}^{N} \mathcal{V}_{i}^{(0)}\right\rangle_{(c)} \\
& \delta_{\xi} \mathcal{Y}_{2}=\frac{1}{2 \pi}\left\langle Q\left(p_{I}\right) \int d^{2} z \xi^{+}(z) \chi_{\bar{z}}^{+}(z) T(z) \prod_{i=1}^{N} \mathcal{V}_{i}^{(0)}\right\rangle_{(c)} \\
& \delta_{\xi} \mathcal{Y}_{3}=\frac{1}{2 \pi} \sum_{i=1}^{N}\left\langle Q\left(p_{I}\right)\left\{\int d^{2} z \delta_{\xi} \chi_{\bar{z}}^{+}(z) S(z) \mathcal{V}_{i}^{(1)}+\int \chi S\left(\delta_{\xi} \mathcal{V}_{i}^{(1)}\right)\right\} \prod_{j \neq i}^{N} \mathcal{V}_{j}^{(0)}\right\rangle \\
& \delta_{\xi} \mathcal{Y}_{4}=\sum_{i \neq j}\left\langle Q\left(p_{I}\right)\left(\delta_{\xi} \mathcal{V}_{i}^{(1)}\right) \mathcal{V}_{j}^{(1)} \prod_{l \neq i, j}^{N} \mathcal{V}_{l}^{(0)}\right\rangle \\
& \delta_{\xi} \mathcal{Y}_{5}=-\sum_{i=1}^{N} \xi^{+} \chi_{\bar{z}}^{+}\left(z_{i}\right)\left\langle Q\left(p_{I}\right) \prod_{j=1}^{N} \mathcal{V}_{j}^{(0)}\right\rangle
\end{aligned}
$$

\subsubsection{Preliminaries}

We begin with the evaluation of $\delta_{\xi} \mathcal{Y}_{1}$. We make use of the following OPEs,

$$
\begin{aligned}
S(z) x_{+}(w) & =-\frac{1}{2} \frac{1}{z-w} \psi_{+}(w) \\
S(z) \psi_{+}(w) & =-\frac{1}{2} \frac{1}{z-w} \partial_{w} x_{+}(w)
\end{aligned}
$$


Of course, $x_{+}(w)$ in the first line is not a well-defined conformal field (but $\partial_{w} x_{+}(w)$ is). The OPE given here is to be understood as used in differences and under derivatives, in which $x_{+}$effectively becomes a conformal field and the OPE is well-defined. The action of the integrated supercurrent on bosonic and fermionic fields is by derivation and therefore fixed by the following elementary rules of operation,

$$
\begin{aligned}
\delta_{\xi}\left\langle\frac{1}{2 \pi} \int \chi S x_{+}(w)\right\rangle_{x} & \equiv \delta_{s s} x_{+}(w)=\xi^{+} \psi_{+}(w) \\
\delta_{\xi}\left\langle\frac{1}{2 \pi} \int \chi S \psi_{+}(w)\right\rangle_{\psi} & \equiv \delta_{s s} \psi_{+}(w)=\xi^{+} \partial_{w} x_{+}(w)
\end{aligned}
$$

Here, we have defined the transformations $\delta_{s s}$ on the fields $x_{+}$and $\psi_{+}$, which act as standard supersymmetry transformations on the matter fields. Using this supersymmetry transformation rule, we may compute the various ingredients needed in evaluating the transformations $\delta_{\xi} \mathcal{Y}$ as follows. First the transformation of the supercurrent itself is needed in $\delta_{\xi} \mathcal{Y}_{1}$ and is given by

$$
\delta_{\xi}\left\langle\frac{1}{2 \pi} \int \chi S S(w)\right\rangle_{(c)} \equiv \delta_{s s} S(w)=\xi^{+} T(w)
$$

Since the double supercurrent insertion occurs in a connected correlator, the stress tensor insertion above will also figure only in a connected correlator, and no self-contractions of $T$ shall occur. One also needs the $\delta_{s s}$ transformations of the vertex operator components. They are given by

$$
\begin{aligned}
\delta_{s s} \mathcal{V}^{(0)}(w) & =-d w \partial_{w}\left(\xi^{+}(w) \epsilon^{\mu} \psi_{+}^{\mu}(w) e^{i k \cdot x_{+}(w)}\right) \\
\delta_{s s} \mathcal{V}^{(1)}(w) & =\frac{1}{2} \xi^{+}(w) \chi_{\bar{w}}{ }^{+}(w) \frac{d \bar{w}}{d w} \mathcal{V}^{(0)}(w) \\
\delta_{s s} \mathcal{V}^{(2)}(w) & =0
\end{aligned}
$$

\subsubsection{Variations of $\mathcal{Y}$}

We are now ready to evaluate all the variations. First, notice that the $\delta_{s s} S(w)$ term generated in $\delta_{\xi} \mathcal{Y}_{1}$ is proportional to the stress tensor insertion and is readily cancelled by $\delta_{\xi} \mathcal{Y}_{2}$. Therefore, it is useful to consider right away the following combinations,

$$
\delta_{\xi} \mathcal{Y}_{1}+\delta_{\xi} \mathcal{Y}_{2}=\frac{1}{2 \pi} \sum_{i=1}^{N}\left\langle Q\left(p_{I}\right) \int \chi S\left(\delta_{s s} \mathcal{V}_{i}^{(0)}\right) \prod_{j \neq i}^{N} \mathcal{V}_{j}^{(0)}\right\rangle_{(c)}
$$

The subscript $(c)$ on the correlator now becomes immaterial since no self-contractions of $S$ can occur, and the subscript will be dropped altogether. 
The contribution of $\delta_{\xi} \mathcal{Y}_{3}$ is evaluated along similar arguments, and we find,

$$
\begin{aligned}
\delta_{\xi} \mathcal{Y}_{3}= & \sum_{i=1}^{N}\left\langle Q\left(p_{I}\right)\left(\delta_{s s} \mathcal{V}_{i}^{(1)}\right) \prod_{j \neq i}^{N} \mathcal{V}_{j}^{(0)}\right\rangle+\sum_{i \neq j}\left\langle Q\left(p_{I}\right)\left(\delta_{s s} \mathcal{V}_{i}^{(0)}\right) \mathcal{V}_{j}^{(1)} \prod_{l \neq i, j}^{N} \mathcal{V}_{l}^{(0)}\right\rangle \\
& +\frac{1}{2 \pi} \sum_{i=1}^{N}\left\langle Q\left(p_{I}\right) \int \chi S\left(\delta_{\xi} \mathcal{V}_{i}^{(1)}\right) \prod_{j \neq i}^{N} \mathcal{V}_{j}^{(0)}\right\rangle
\end{aligned}
$$

The $\delta_{\xi}$ variation of $\mathcal{V}_{i}^{(1)}$ in the last term of (5.19) and the $\delta_{s s}$ variation of $\mathcal{V}_{i}^{(0)}$ of (5.18) partially combine into an exact differential, as follows,

$$
\begin{aligned}
\delta_{\xi} \mathcal{V}^{(1)}(w)+\delta_{s s} \mathcal{V}^{(0)}(w)= & -d_{w}\left(\xi^{+}(w) \epsilon^{\mu} \psi_{+}^{\mu}(w) e^{i k \cdot x_{+}(w)}\right) \\
& +d \bar{w} \xi^{+}(w) \partial_{\bar{w}}\left(\epsilon^{\mu} \psi_{+}^{\mu}(w) e^{i k \cdot x_{+}(w)}\right)
\end{aligned}
$$

The insertion of the last term above into the last term of (5.19) is given by

$$
\frac{1}{2 \pi} \sum_{i=1}^{N}\left\langle Q\left(p_{I}\right) \int \chi S d \bar{z}_{i} \xi^{+}\left(z_{i}\right) \partial_{\bar{z}_{i}}\left(\epsilon^{\mu} \psi_{+}^{\mu}\left(z_{i}\right) e^{i k_{i} \cdot x_{+}\left(z_{i}\right)}\right) \prod_{j \neq i}^{N} \mathcal{V}_{j}^{(0)}\right\rangle
$$

The presence of the $\partial_{\bar{z}_{i}}$ derivative guarantees that its argument will only give non-vanishing contractions with the supercurrent (as the points $z_{j}$ are all distinct from $z_{i}$ for $j \neq i$ ). These contractions may be computed,

$$
\begin{aligned}
\left\langle S(w) \partial_{\bar{z}_{i}} x_{+}\left(z_{i}\right)\right\rangle_{*} & =-\pi \delta\left(w, z_{i}\right) \psi_{+}^{\mu}\left(z_{i}\right) \\
\left\langle S(w) \partial_{\bar{z}_{i}} \psi_{+}\left(z_{i}\right)\right\rangle_{*} & =-\pi \delta\left(w, z_{i}\right) \partial_{z_{i}} x_{+}^{\mu}\left(z_{i}\right)
\end{aligned}
$$

and as a result,

$$
\left\langle S(w) \partial_{\bar{z}_{i}}\left(\epsilon^{\mu} \psi_{+}^{\mu}\left(z_{i}\right) e^{i k_{i} \cdot x_{+}\left(z_{i}\right)}\right)\right\rangle_{*}=-\pi \delta\left(w, z_{i}\right) \mathcal{V}_{i}^{(0)}\left(z_{i}\right)
$$

Combining all, we have

$$
\begin{aligned}
\sum_{a=1}^{3} \delta_{\xi} \mathcal{Y}_{a}= & \sum_{i=1}^{N}\left\langle Q\left(p_{I}\right)\left(\delta_{s s} \mathcal{V}_{i}^{(1)}\right) \prod_{j \neq i}^{N} \mathcal{V}_{j}^{(0)}\right\rangle+\frac{1}{2} \sum_{i=1}^{N} \xi^{+} \chi_{\bar{z}}^{+}\left(z_{i}\right)\left\langle Q\left(p_{I}\right) \prod_{j=1}^{N} \mathcal{V}_{j}^{(0)}\right\rangle \\
& +\sum_{i \neq j}\left\langle Q\left(p_{I}\right)\left(\delta_{s s} \mathcal{V}_{i}^{(0)}\right) \mathcal{V}_{j}^{(1)} \prod_{l \neq i, j}^{N} \mathcal{V}_{l}^{(0)}\right\rangle+\sum_{i=1}^{N} d_{i} \mathcal{R}_{i}^{\xi(1)}[\delta]
\end{aligned}
$$

where the exact differential term is given by

$$
\mathcal{R}_{i}^{\xi(1)}[\delta]=\frac{1}{2 \pi}\left\langle Q\left(p_{I}\right) \int \chi S \epsilon_{i}^{\mu} \xi^{+}\left(z_{i}\right) \psi_{+}^{\mu}\left(z_{i}\right) e^{i k_{i} \cdot x_{+}\left(z_{i}\right)} \prod_{j \neq i} \mathcal{V}_{i}^{(0)}\right\rangle
$$


The next step is to notice that the first and last terms in (5.24) cancel $\delta_{\xi} \mathcal{Y}_{5}$ completely. Thus, we are left with

$$
\sum_{a=1}^{5} \delta_{\xi} \mathcal{Y}_{a}=\sum_{i \neq j}\left\langle Q\left(p_{I}\right)\left(\delta_{s s} \mathcal{V}_{i}^{(0)}+\delta_{\xi} \mathcal{V}_{i}^{(1)}\right) \mathcal{V}_{j}^{(1)} \prod_{l \neq i, j}^{N} \mathcal{V}_{l}^{(0)}\right\rangle+\sum_{i=1}^{N} d_{i} \mathcal{R}_{i}^{\xi(1)}[\delta]
$$

We use again (5.20) to combine the two variations in the parentheses into an exact differential and a remainder, which involves the operator $\partial_{\bar{z}_{i}}\left(\epsilon_{i}^{\mu} \psi_{+}^{\mu}\left(z_{i}\right) e^{i k_{i} \cdot x_{+}\left(z_{i}\right)}\right)$, whose correlators vanish because of the $\partial_{\bar{z}_{i}}$ operator acting on a holomorphic expression for separated points $z_{j}$. We are left with

$$
\sum_{a=1}^{5} \delta_{\xi} \mathcal{Y}_{a}=\sum_{i=1}^{N} d_{i}\left(\mathcal{R}_{i}^{\xi(1)}[\delta]+\mathcal{R}_{i}^{\xi(2)}[\delta]\right)
$$

where the new exact differential term is given by

$$
\mathcal{R}_{i}^{\xi(2)}[\delta]=-\sum_{j \neq i}\left\langle Q\left(p_{I}\right) \xi^{+}\left(z_{i}\right) \epsilon_{i}^{\mu} \psi_{+}^{\mu}\left(z_{i}\right) e^{i k_{i} \cdot x_{+}\left(z_{i}\right)} \mathcal{V}_{j}^{(1)} \prod_{l \neq i, j} \mathcal{V}_{l}^{(0)}\right\rangle
$$

\subsubsection{Structure of the Exact Differential Terms}

Using this last result, we have

$$
\delta_{\xi} \mathcal{B}[\delta]^{(c)}=d \mu_{0}[\delta] \sum_{a=1}^{5} \delta_{\xi} \mathcal{Y}_{a}=\sum_{i=1}^{N} d_{i} \mathcal{R}_{i}^{\xi}[\delta]
$$

Here, $\mathcal{R}_{i}^{\xi}[\delta]=\mathcal{R}_{i}^{\xi(1)}[\delta]+\mathcal{R}_{i}^{\xi(2)}[\delta]$ is given succinctly by the following expression,

$$
\mathcal{R}_{i}^{\xi}[\delta]=\left\langle Q\left(p_{I}\right) \varnothing_{i}\left(\frac{1}{2 \pi} \int \chi S \prod_{l \neq i} \mathcal{V}_{l}^{(0)}+\sum_{j \neq i} \mathcal{V}_{j}^{(1)} \prod_{l \neq i, j} \mathcal{V}_{l}^{(0)}\right)\right\rangle
$$

where we have defined the operator

$$
\varnothing_{i} \equiv-d \mu_{0}[\delta] \xi^{+}\left(z_{i}\right) \epsilon_{i}^{\mu} \psi_{+}^{\mu}\left(z_{i}\right) e^{i k_{i} \cdot x_{+}\left(z_{i}\right)}
$$

This form has the following properties

- $\mathcal{R}_{i}^{\xi}[\delta]$ is a form of weight $(0,0)$ in $z_{i}$;

- $\mathcal{R}_{i}^{\xi}[\delta]$ is a form of weight $(1,0) \oplus(0,1)$ in each $z_{j}$, with $j \neq i$;

- $\mathcal{R}_{i}^{\xi}[\delta]$ is closed in each $z_{j}$ for $j \neq i$, i.e. $d_{j} \mathcal{R}_{i}^{\xi}[\delta]=0$. 
Only the last assertion requires a proof. We calculate $d_{j} \mathcal{R}_{i}^{\xi}[\delta]$ for $j \neq i$.

The first term in (5.30) is a form of weight $(1,0)$ in $z_{j}$, and thus only the $d \bar{z}_{j} \partial_{\bar{z}_{j}}$ part of the differential $d_{j}$ acts on this term. The only contributions come from the further contractions of this derivative with the supercurrent.

The second term in (5.30) is a sum of terms of two types. The first type consists of those terms involving the form $\mathcal{V}_{l}^{(1)}\left(z_{l}\right)$ with $l \neq j$; these are forms of weight $(1,0)$ in $z_{j}$, and thus only the $d \bar{z}_{j} \partial_{\bar{z}_{j}}$ part of the differential $d_{j}$ acts. Since, for distinct points $z_{l}$, the argument is holomorphic, this type of terms automatically contributes 0 .

The second type consists of those terms involving the form $\mathcal{V}_{j}^{(1)}\left(z_{j}\right)$; this form is of weight $(0,1)$ in $z_{j}$ and thus only the $d z_{j} \partial_{z_{j}}$ part of the differential $d_{j}$ acts. Putting all together, we have

$$
d_{j} \mathcal{R}_{i}^{\xi}[\delta]=\left\langle Q\left(p_{I}\right) \varnothing_{i}\left(\frac{1}{2 \pi} \int \chi S d \bar{z}_{j} \partial_{\bar{z}_{j}} \mathcal{V}_{j}^{(0)}+d z_{j} \partial_{z_{j}} \mathcal{V}_{j}^{(1)}\right) \prod_{l \neq i, j} \mathcal{V}_{l}^{(0)}\right\rangle
$$

Using the following contraction,

$$
\left\langle\frac{1}{2 \pi} \int \chi S d \bar{z}_{j} \partial_{\bar{z}_{j}} \mathcal{V}_{j}^{(0)}\right\rangle_{*}=-d z_{j} \partial_{z_{j}}\left(\mathcal{V}_{j}^{(1)}\right)
$$

Adding both contributions, we find that

$$
d_{j} \mathcal{R}_{i}^{\xi}[\delta]=0 \quad j \neq i
$$

which completes the proof. 


\section{Slice-Independence of the Full Amplitudes}

In this section, we use the closedness of the chiral amplitudes and exactness of their variations induced by gauge slice changes proven in the preceding two sections to establish the gauge slice independence of the GSO projected, vertex integrated, superstring scattering amplitudes.

The chiral blocks $\mathcal{B}[\delta]$ have non-trivial monodromy as each insertion point $z_{i}$ is transported around a $B$-cycle (but no monodromy around an $A$-cycle) on the worldsheet. This monodromy is due to the monodromy of the effective bosonic propagator $\left\langle x_{+}(z) x_{+}(w)\right\rangle=$ $-\ln E(z, w)$,

$$
-\ln E\left(z+B_{K}, w\right)=-\ln E(z, w)+\pi i \Omega_{K K}+2 \pi i \int_{w}^{z} \omega_{K}
$$

The fermionic propagator $S_{\delta}(z, w)$ has no monodromy (for even spin structures). Carrying out the contractions in $\mathcal{B}[\delta]$, we find that $\mathcal{B}[\delta]$ has the monodromy indicated in (1.8) (see [6], eq. (5.46)). Formally, this result is readily seen if we note that the internal and external momenta-dependence of $\mathcal{B}[\delta]$ comes from the following insertion (c.f. (2.8) )

$$
Q\left(p_{I}\right) W(z, \theta ; \epsilon, k)=\exp \left(i p_{I}^{\mu} \oint_{B_{I}} d z \partial_{z} x_{+}^{\mu}+\left(i k^{\mu}+\epsilon^{\mu} \theta \partial_{z}\right) x_{+}^{\mu}+\left(i k^{\mu} \theta+\epsilon^{\mu}\right) \psi_{+}^{\mu}\right)
$$

Here we have set $\left(z_{1}, \theta_{1}\right)=(z, \theta)$ and suppressed the explicit dependence on the other variables, such as $\chi$ and $\hat{\mu}$ for notational simplicity. Transporting $z$ along a $B_{K}$ cycle results in a shift of the exponential by

$$
\left(i k^{\mu}+\epsilon^{\mu} \theta \partial_{z}\right) \oint_{B_{K}} d z \partial_{z} x_{+}^{\mu}=i k^{\mu} \oint_{B_{K}} d z \partial_{z} x_{+}^{\mu}
$$

which is exactly the same as a shift of $p_{I}$ by $k^{\mu}$. Applying the same arguments to the variations of $\mathcal{B}[\delta]$ obtained in section $\S 3$, we readily see that the forms $\mathcal{R}_{i}^{v}[\delta]$ and $\mathcal{R}_{i}^{\xi}[\delta]$ induced by changes of $\hat{\mu}$ Beltrami differential and by gauge slice $\chi_{\alpha}$ have the same monodromy (1.8) as $\mathcal{B}[\delta]$.

We can give now the proof of gauge slice independence. Consider first the $\chi$-independence. In the preceding sections, we have proven the following equations,

$$
\begin{array}{rlr}
\delta_{\xi} \mathcal{B}[\delta]\left(z ; \epsilon, k, p_{I}\right) & =\sum_{i=1}^{N} d_{i} \mathcal{R}_{i}^{\xi}[\delta]\left(z ; \epsilon, k, p_{I}\right) \\
\delta_{\bar{\xi}} \mathcal{B}[\delta]\left(z ; \epsilon, k, p_{I}\right) & =0 & \\
d_{i} \mathcal{B}[\delta]\left(z ; \epsilon, k, p_{I}\right) & =0 & j \neq i \\
d_{j} \mathcal{R}_{i}^{\xi}[\delta]\left(z ; \epsilon, k, p_{I}\right) & =0
\end{array}
$$


Here, $d_{i}$ denotes the total differential in the variable $z_{i}$. Furthermore, $\xi$ denotes the $\xi^{+}$ component of the change of spinor fields and $\bar{\xi}$ denotes its complex conjugate. Now the GSO projected, vertex integrated, superstring scattering amplitudes is defined by

$$
\sum_{\delta, \bar{\delta}} \mathcal{C}_{\delta, \bar{\delta}} \int d p_{I} \int_{\Sigma^{N}} \mathcal{B}[\delta]\left(z ; \epsilon, k, p_{I}\right) \wedge \overline{\mathcal{B}}[\bar{\delta}]^{\prime}\left(\bar{z} ; \bar{\epsilon}, k, p_{I}\right)
$$

Here, the left and right moving strings do not necessarily have to be the same (although they are required to have the same monodromy), a fact that is indicated by a prime on $\overline{\mathcal{B}}$. The coefficients $C_{\delta, \bar{\delta}}$ govern the GSO projection prescription and are independent of the moduli $\Omega$ and of the insertion points $z_{1}, \cdots, z_{N}$. Under a change $\delta_{\xi} \chi_{\bar{z}}{ }^{+}(z)=-2 \partial_{\bar{z}} \xi^{+}(z)$ of gauge slice, the contribution to the above amplitude from each pair of spin structures $\delta, \bar{\delta}$ changes to

$$
\begin{gathered}
\int d p_{I} \int_{\Sigma^{N}}\left(\mathcal{B}[\delta]+\sum_{i=1}^{N} d_{i} \mathcal{R}_{i}^{\xi}[\delta]\right) \wedge\left(\overline{\mathcal{B}}[\bar{\delta}]^{\prime}+\sum_{j=1}^{N} d_{j} \overline{\mathcal{R}}_{j}^{\xi}[\delta]^{\prime}\right) \\
=\int d p_{I} \int_{\Sigma^{N}}\left(\mathcal{B}[\delta] \wedge \overline{\mathcal{B}}[\bar{\delta}]^{\prime}+\sum_{i=1}^{N} d_{i}\left(\mathcal{R}_{i}^{\xi}[\delta] \wedge \overline{\mathcal{B}}[\bar{\delta}]^{\prime}\right)+\sum_{j=1}^{N} d_{j}\left((-)^{N} \mathcal{B}[\delta] \wedge \mathcal{R}_{j}^{\xi}[\delta]^{\prime}\right)\right. \\
\left.+\sum_{i, j=1}^{N} d_{i}\left(\mathcal{R}_{i}^{\xi}[\delta] \wedge d_{j} \overline{\mathcal{R}}_{j}^{\xi}[\bar{\delta}]^{\prime}\right)\right)
\end{gathered}
$$

where we have used the fact that $\mathcal{B}[\delta], \overline{\mathcal{B}}[\bar{\delta}]^{\prime}$ are closed 1 -forms in all variables, while $\mathcal{R}_{i}^{\xi}[\delta]$, $\overline{\mathcal{R}}_{i}^{\bar{\xi}^{\prime}}$ are closed 1 -forms in all variables except $z_{i}$. Isolating the integration over $z_{i}$, we note that the object $\mathcal{R}_{i}^{\xi}[\delta] \wedge \overline{\mathcal{B}}[\bar{\delta}]^{\prime}$ is a 1 -form in $z_{i}$ with the monodromy (1.8). Its integral must then vanish by the Riemann identity, upon integration over the internal loop momenta $p_{I}$. More precisely, the surface $\Sigma$ can be cut apart along its homology cycles into a simplyconnected region with boundary $\prod_{K=1}^{2} A_{K} B_{K} A_{K}^{-1} B_{K}^{-1}$. Applying Stokes' theorem to the integral of $d_{i}\left(\mathcal{R}_{i}^{\xi}[\delta] \wedge \overline{\mathcal{B}}[\bar{\delta}]^{\prime}\right)$ over this region, we obtain

$$
\begin{aligned}
\int_{\Sigma} d_{i}\left(\mathcal{R}_{i}^{\xi}[\delta] \wedge \overline{\mathcal{B}}[\bar{\delta}]^{\prime}\right) & =\sum_{K=1}^{2} \oint_{B_{K}}\left(\left(\mathcal{R}_{i}^{\xi}[\delta] \wedge \overline{\mathcal{B}}[\bar{\delta}]^{\prime}\right)\left(z_{i}+A_{K} ; p_{I}\right)-\left(\mathcal{R}_{i}^{\xi}[\delta] \wedge \overline{\mathcal{B}}[\bar{\delta}]^{\prime}\right)\left(z_{i} ; p_{I}\right)\right) \\
& -\sum_{K=1}^{2} \oint_{A_{K}}\left(\left(\mathcal{R}_{i}^{\xi}[\delta] \wedge \overline{\mathcal{B}}[\bar{\delta}]^{\prime}\right)\left(z_{i}+B_{K} ; p_{I}\right)-\left(\mathcal{R}_{i}^{\xi}[\delta] \wedge \overline{\mathcal{B}}[\bar{\delta}]^{\prime}\right)\left(z_{i} ; p_{I}\right)\right)
\end{aligned}
$$

Here we have indicated the dependence on $p_{I}$ because it plays an important role. In view of the monodromy (1.8) for $\mathcal{R}_{i}^{\xi}[\delta] \wedge \overline{\mathcal{B}}[\bar{\delta}]^{\prime}$, the above expression reduces to

$$
-\sum_{K=1}^{2} \oint_{A_{K}}\left(\left(\mathcal{R}_{i}^{\xi}[\delta] \wedge \overline{\mathcal{B}}[\bar{\delta}]^{\prime}\right)\left(z ; p_{I}+\delta_{I K} k_{i}\right)-\left(\mathcal{R}_{i}^{\xi}[\delta] \wedge \overline{\mathcal{B}}[\bar{\delta}]^{\prime}\right)\left(z ; p_{I}\right)\right)
$$


For fixed $p_{I}$, this may not vanish, but its integral over $p_{I}$ does vanish by translation invariance of the measure $d p_{I}$ and the integration region of $p_{I}$,

$$
\int d p_{I} \sum_{K=1}^{2} \oint_{A_{K}}\left(\left(\mathcal{R}_{i}^{\xi} \wedge \overline{\mathcal{B}}[\bar{\delta}]^{\prime}\right)\left(z ; p_{I}^{\mu}+\delta_{I K} k_{i}^{\mu}\right)-\left(\mathcal{R}_{i}^{\xi} \wedge \overline{\mathcal{B}}[\bar{\delta}]^{\prime}\right)\left(z ; p_{I}^{\mu}\right)\right)=0
$$

Thus the contribution of the term $d_{i}\left(\mathcal{R}_{i}^{\xi}[\delta] \wedge \overline{\mathcal{B}}[\bar{\delta}]^{\prime}\right)$ is 0 . The contributions of the other exact differentials in (6.6) vanish by the same argument, establishing the desired invariance of the integrated superstring amplitudes under changes of $\chi$ slice. The situation for changes of $\hat{\mu}$ slices is similar, starting from the basic properties

$$
\begin{array}{rlr}
\delta_{v} \mathcal{B}[\delta]\left(z ; \epsilon, k, p_{I}\right) & =\sum_{i=1}^{N} d_{i} \mathcal{R}_{i}^{v}[\delta]\left(z ; \epsilon, k, p_{I}\right) \\
\delta_{\bar{v}} \mathcal{B}[\delta]\left(z ; \epsilon, k, p_{I}\right) & =0 & \\
d_{j} \mathcal{R}_{i}^{v}[\delta]\left(z ; \epsilon, k, p_{I}\right) & =0 & j \neq i
\end{array}
$$

The proof of gauge invariance under changes of both $\chi_{\alpha}$ and $\hat{\mu}$ slices is now complete. 


\section{References}

[1] E. D'Hoker and D.H. Phong, "Two-Loop Superstrings I, Main Formulas", Phys. Lett. B529 (2002) 241-255; hep-th/0110247.

[2] E. D'Hoker and D.H. Phong, "Two-Loop Superstrings II, The chiral Measure on Moduli Space", Nucl. Phys. B636 (2002) 3-60; hep-th/0110283

[3] E. D'Hoker and D.H. Phong, "Two-Loop Superstrings III, Slice Independence and Absence of Ambiguities", Nucl. Phys. B636 (2002) 61-79; hep-th/0111016.

[4] E. D'Hoker and D.H. Phong, “Two-Loop Superstrings IV, The Cosmological Constant and Modular Forms", Nucl. Phys. B639 (2002) 129-181; hep-th/0111040

[5] E. D'Hoker and D.H. Phong, "Lectures on two-loop superstrings", Hangzhou, Beijing 2002, hep-th/0211111.

[6] E. D'Hoker and D.H. Phong, "Conformal scalar fields and chiral splitting on super Riemann surfaces", Commun. Math. Physics 125 (1989) 469-513.

[7] E. D'Hoker and D.H. Phong, "The geometry of string perturbation theory", Rev. Modern Physics 60 (1988) 917-1065.

[8] E. D'Hoker and D.H. Phong, "Two-Loop Superstrings VI, Non-renormalization Theorems and the 4-Point Function", (2005).

[9] E. D'Hoker and D.H. Phong, "Two-Loop Superstrings VII, Holomorphic Blocks", in preparation.

[10] E. Verlinde and H. Verlinde, "Multiloop calculations in covariant superstring theory", Phys. Lett. B192 (1987) 95-102;

E. Verlinde and H. Verlinde, "Superstring Perturbation Theory", In Superstrings 88, M. Green et al. Eds., World Scientific Publ. Co. Singapore (1989) 222-240;

H. Verlinde, "A note on the integral over fermionic supermoduli", Utrecht Preprint No. THU-87/26 (1987) unpublished.

[11] E. Martinec, "Non-renormalization Theorems and Fermionic String Finiteness", Phys. Lett. B171 (1986) 189;

G. Moore, J. Harris, P. Nelson, and I.M. Singer, "Modular forms and the cosmological constant", Phys. Lett. B 178 (1986) 167-173;

S. Chaudhuri, H. Kawai and S. H. H. Tye, "Path Integral Formulation Of Closed Strings," Phys. Rev. D 36, 1148 (1987);

J. Atick, J. Rabin, and A. Sen, "An ambiguity in fermionic string theory", Nucl. Phys. B 299 (1988) 279-294;

J. Atick, G. Moore, and A. Sen, "Some global issues in string perturbation theory", Nucl. Phys. B 308 (1988) 1; "Catoptric tadpoles", Nucl. Phys. B 307 (1988) 221-273; 
H. La and P. Nelson, "Unambiguous fermionic string amplitudes", Phys. Rev. Lett. 63 (1989) 24-27;

M. Martellini, P. Teofilato, "Global structure of the superstring partition function and resolution of the supermoduli measure ambiguity", Phys. Lett. B 211 (1988) 293.

[12] L. Alvarez-Gaumé, C. Gomez, G. Moore, P. Nelson, and C. Vafa, "Fermionic strings in the operator formalism", Nucl. Phys. B 311 (1988) 333;

A. Neveu and P. West, "Group theoretic approach to the superstring and its supermoduli" Nucl. Phys. B 311 (1988) 79.

P. Di Vecchia, K. Hornfeck, M. Frau, A. Lerda, S. Sciuto, " $N$ string, $G$ loop vertex for the fermionic string, Phys. Lett. B211 (1988) 301;

O. Lechtenfeld, "On Finiteness of the Superstring", Nucl. Phys. B 322 (1989) 82;

O. Yasuda, "Multiloop Modular Invariance of D=10 Type II Superstring Theory", Nucl. Phys. B318 (1989) 397;

O. Lechtenfeld and A. Parkes, "On covariant multiloop superstring amplitudes", Nucl. Phys.

B 332 (1990) 39-82;

O. Lechtenfeld, "Factorization and modular invariance of multiloop superstring amplitudes in the unitary gauge", Nucl. Phys. B 338 (1990) 403-414;

[13] O. Lechtenfeld and A. Parkes, "On the Vanishing of the genus 2 Superstring Vacuum Amplitude", Phys. Lett. B 202 (1988) 75;

E. Gava and R. Iengo, "Modular Invariance and the Two Loop Vanishing of the Cosmological Constant", Phys. Lett. B 207 (1988) 283;

R. Iengo and C.J. Zhu, "Notes on Non-renormalization Theorem in Superstring Theories", Phys. Lett. B 212 (1988) 309;

G. Moore and A. Morozov, "Some remarks on two-loop string calculations", Nucl. Phys. B 306 (1988) 387-404;

A. Morozov, "Pointwise Vanishing of Two-Loop Contributions to 1, 2, 3 Point Functions in Superstring Theories", Nucl. Phys. B 318 (1989) 137;

[14] A. Morozov, "On the two-loop contribution to the superstring four-point function", Phys. Lett. B 209 (1988) 473-476;

O. Yasuda, "Factorization of a two loop Four Point Superstring Amplitude", Phys. Rev. Lett. 60 (1988) 1688; erratum-ibid 61 (1988) 1678;

R. Iengo and C.J. Zhu, "Two-loop computation of the four-particle in heterotic string theory", Phys. Lett. B 212 (1988) 313;

R. Iengo and C.J. Zhu, "Explicit modular invariant two-loop superstring amplitude relevant to $R^{4}$, JHEP 06 (1999) 011.

[15] Z. J. Zheng, J. B. Wu and C. J. Zhu, "Two-loop superstrings in hyperelliptic language. I: The main results," Phys. Lett. B 559, 89 (2003) arXiv:hep-th/0212191;

Z. J. Zheng, J. B. Wu and C. J. Zhu, "Two-loop superstrings in hyperelliptic language. II: The vanishing of the cosmological constant and the non-renormalization theorem," Nucl. Phys. B 663, 79 (2003) arXiv:hep-th/0212198;

Z. J. Zheng, J. B. Wu and C. J. Zhu, "Two-loop superstrings in hyperelliptic language. III: 
The four-particle amplitude," Nucl. Phys. B 663, 95 (2003) arXiv:hep-th/0212219;

C. J. Zhu, "Two-loop computation in superstring theory," arXiv:hep-th/0301018

J. B. Wu and C. J. Zhu, "Comments on two-loop four-particle amplitude in superstring theory," JHEP 0305, 056 (2003) arXiv:hep-th/0303152.

[16] S. Mandelstam, "The $\mathrm{n}$ loop string amplitude: Explicit formulas, finiteness and absence of ambiguities," Phys. Lett. B 277, 82 (1992);

N. Berkovits, "Lorentz covariant Green-Schwarz superstring amplitudes," Phys. Lett. B 300, 53 (1993) arXiv:hep-th/9211025.

[17] N. Berkovits, "Multiloop amplitudes and vanishing theorems using the pure spinor formalism for the superstring," arXiv:hep-th/0406055.

N. Berkovits, "Introduction to superstring theory," Prepared for 11th Jorge Andre Swieca Summer School on Particle and Fields, Campos do Jordao, Brazil, 14-27 Jan 2001;

N. Berkovits, "Covariant multiloop superstring amplitudes," arXiv:hep-th/0410079

N. Berkovits and D. Z. Marchioro, "Relating the Green-Schwarz and pure spinor formalisms for the superstring," arXiv:hep-th/0412198.

[18] P.S. Howe, "Super Weyl transformations in two dimensions", J. Phys. A (1979) 393;

E. Martinec, "Superspace geometry of superstrings", Phys. Rev. D 28 (1983) 2604;

E. D'Hoker and D.H. Phong, "Superholomorphic anomalies and supermoduli space", Nucl. Phys. B 292 (1987) 317.

S. Giddings and P. Nelson, "The geometry of super Riemann surfaces", Commun. Math. Phys. 116 (1988), 607-634.

[19] E. D'Hoker and D.H. Phong, "Vertex Operators for Closed Strings", Phys. Rev D35 (1987) 3890 .

[20] E. D'Hoker and D.H. Phong, "Superstrings, super Riemann surfaces, and supermoduli space", Contribution to the Proc. of the 'String Theory' Conference, Rome, Italy, June 1988, published in Rome String Theory 1988: 17-68 (QCD162:S75:1988)

[21] K. Aoki, E. D'Hoker and D.H. Phong, "Unitarity of Closed Superstring Perturbation Theory", Nucl. Phys. B342 (1990) 149.

[22] K. Peeters, P. Vanhove and A. Westerberg, "Chiral splitting and world-sheet gravitinos in higher-derivative string amplitudes," Class. Quant. Grav. 19, 2699 (2002) arXiv:hep-th/0112157.

[23] D. Friedan, E. Martinec, and S. Shenker, "Conformal invariance, supersymmetry, and string theory", Nucl. Phys. B 271 (1986) 93;

E. D'Hoker and D.H. Phong, "Loop amplitudes for the fermionic string", Nucl. Phys. B 278 (1986) 225;

G. Moore, P. Nelson, and J. Polchinski, "Strings and supermoduli", Phys. Lett. B 169 (1986) 47-53. 